\title{
Odd dimensional analogue of the Euler characteristic
}

\author{
L. Borsten, ${ }^{a}$ M.J. Duff ${ }^{b, c}$ and S. Nagy ${ }^{d}$ \\ ${ }^{a}$ Maxwell Institute and Department of Mathematics, Heriot-Watt University, \\ Edinburgh EH14 4 AS, U.K. \\ ${ }^{b}$ Institute for Quantum Science and Engineering and Hagler Institute for Advanced Study, \\ Texas A\&M University, \\ College Station, TX, 77840, U.S.A. \\ ${ }^{c}$ Theoretical Physics, Blackett Laboratory, Imperial College London, \\ Prince Consort Road, London SW7 2AZ, U.K. \\ ${ }^{d}$ Centre for Theoretical Physics, Department of Physics and Astronomy, \\ Queen Mary University of London, \\ 327 Mile End Road, London E1 4NS, U.K.
}

E-mail: 1.borsten@hw.ac.uk, m.duff@imperial.ac.uk, s.nagy@qmul.ac.uk

Abstract: When compact manifolds $X$ and $Y$ are both even dimensional, their Euler characteristics obey the Künneth formula $\chi(X \times Y)=\chi(X) \chi(Y)$. In terms of the Betti numbers $b_{p}(X), \chi(X)=\sum_{p}(-1)^{p} b_{p}(X)$, implying that $\chi(X)=0$ when $X$ is odd dimensional. We seek a linear combination of Betti numbers, called $\rho$, that obeys an analogous formula $\rho(X \times Y)=\chi(X) \rho(Y)$ when $Y$ is odd dimensional. The unique solution is $\rho(Y)=-\sum_{p}(-1)^{p} p b_{p}(Y)$. Physical applications include: $(1) \rho \rightarrow(-1)^{m} \rho$ under a generalized mirror map in $d=2 m+1$ dimensions, in analogy with $\chi \rightarrow(-1)^{m} \chi$ in $d=2 m$; (2) $\rho$ appears naturally in compactifications of M-theory. For example, the 4-dimensional Weyl anomaly for M-theory on $X^{4} \times Y^{7}$ is given by $\chi\left(X^{4}\right) \rho\left(Y^{7}\right)=\rho\left(X^{4} \times Y^{7}\right)$ and hence vanishes when $Y^{7}$ is self-mirror. Since, in particular, $\rho\left(Y \times S^{1}\right)=\chi(Y)$, this is consistent with the corresponding anomaly for Type IIA on $X^{4} \times Y^{6}$, given by $\chi\left(X^{4}\right) \chi\left(Y^{6}\right)=\chi\left(X^{4} \times Y^{6}\right)$, which vanishes when $Y^{6}$ is self-mirror; (3) In the partition function of $p$-form gauge fields, $\rho$ appears in odd dimensions as $\chi$ does in even.

KEywords: Anomalies in Field and String Theories, M-Theory, BRST Quantization ArXiv EPrint: 2105.13268 


\section{Contents}

1 Introduction 1

2 The $\rho$-characteristic $\quad 4$

2.1 Definition 4

2.2 Uniqueness 6

2.3 Cosets 7

3 Mirror symmetry $\quad 9$

3.1 Conventional mirror symmetry: Calabi-Yau and Joyce 9

3.2 Generalised mirror maps; $\chi \rightarrow \pm \chi$ in even $d \quad 11$

3.3 Generalised mirror maps; $\rho \rightarrow \pm \rho$ in odd $d \quad 16$

$\begin{array}{llr}4 & \text { Weyl anomalies } & 17\end{array}$

4.1 Weyl anomalies 17

$4.2 D=10$ type IIA and $D=11$ M-theory: the roles of $\chi$ and $\rho \quad 18$

4.3 Special case $X^{d}=X^{d-1} \times S^{1} ; \rho\left(X^{d}\right)=\chi\left(X^{d-1}\right)$

5 Partition functions $\quad 20$

5.1 Topological quantum field theory 20

5.2 Duality anomalies 22

5.2.1 Electromagnetic duality and the $\chi, \rho$ characteristics 22

5.2.2 Massive duality anomalies and Kaluza-Klein compactification 26

$\begin{array}{llr}6 & \text { Conclusions } & 29\end{array}$

A $B_{4}$ coefficients for $p$-forms

\section{Introduction}

The familiar Euler characteristic of a manifold $X$, given by the alternating sum of Betti numbers $b_{p}(X)$,

$$
\chi(X)=\sum_{p=0}^{d}(-1)^{p} b_{p}(X)
$$

is identically zero when $d=\operatorname{dim} X=2 m+1$. In this paper we argue that in several respects the topological invariant

$$
\rho(X)=-\sum_{p=0}^{d}(-1)^{p} p b_{p}(X)
$$

is a natural generalisation of the Euler characteristic that is non-trivial in odd dimensions: 
(i) Kunneth formula (section 2): when closed manifolds $X$ and $Y$ are both even dimensional, their Euler characteristics obey the non-trivial ${ }^{1}$ Künneth formula

$$
\chi(X \times Y)=\chi(X) \chi(Y)
$$

whereas $\rho$ obeys an analogous formula when $\operatorname{dim} Y$ is odd

$$
\rho(X \times Y)=\chi(X) \rho(Y) .
$$

Indeed it is the unique (up to trivial scaling and shifts, cf. section 2) linear combination of Betti numbers to do so.

(ii) Special case $Y^{(d+1)}=X^{d} \times S^{1}$ (section 2): in this case

$$
\rho(Y)=\chi(X) .
$$

(iii) Generalised mirror map (section 3): for $d=2 m$, there is a mirror map under which

$$
\chi(X) \rightarrow(-1)^{m} \chi(X)
$$

For $d=2 m+1$, there is a mirror map under which

$$
\rho(Y) \rightarrow(-1)^{m} \rho(Y)
$$

(iv) Weyl anomalies in Type IIA and M-theory compactifications (section 4): the topological invariant $\rho$ first made its appearance in the case $d=7$ corresponding to a compactification of M-theory from $D=11$ to $D=4$ spacetime dimensions $^{2}$ [1-3], as we now recall in table 1.

For $d=10$ Type IIA on $X^{4}($ spacetime $) \times X^{6}($ internal $)$ the $d=4$ on-shell Weyl anomaly $\mathcal{A}^{\mathrm{W}}$ is given by ${ }^{3}$

$$
\int \mathcal{A}^{\mathrm{W}}=-\frac{1}{24} \chi\left(X^{4}\right) \chi\left(X^{6}\right)=-\frac{1}{24} \chi\left(X^{4} \times X^{6}\right)
$$

on using the Künneth rule (1.3) and hence vanishes when $X^{6}$ (and therefore $X^{10}$ ) is self-mirror.

For $d=11$ M-theory on $X^{4}$ (spacetime $) \times Y^{7}($ internal $)$ the $d=4$ on-shell Weyl anomaly $\mathcal{A}^{\mathrm{W}}$ is given by

$$
\int \mathcal{A}^{\mathrm{W}}=-\frac{1}{24} \chi\left(X^{4}\right) \rho\left(Y^{7}\right)=-\frac{1}{24} \rho\left(X^{4} \times Y^{7}\right)
$$

on using the Künneth rule (1.4) and hence vanishes when $Y^{7}$ (and therefore $X^{11}$ ) is self-mirror.

By virtue of the special case (1.5), this is entirely consistent with the equivalence

$$
\text { M-theory on } X^{10} \times S^{1} \equiv \text { IIA on } X^{10} \text {. }
$$

\footnotetext{
${ }^{1}$ Of course, it is satisfied trivially when at least one manifold is odd dimensional.

${ }^{2}$ Note, we use $D(d)$ to refer to the dimension of a Lorentzian (Riemannian) manifold.

${ }^{3}$ Where it is understood that we Wick rotate the spacetime manifold to be Euclidean and assume it is closed.
} 


\begin{tabular}{|c|c|c|c|c|c|}
\hline$D=11$ & $D=4$ & $f(\phi)$ & $360 A(\phi)$ & Multiplicity for $Y^{7}$ & Multiplicity for $X^{6} \times S^{1}$ \\
\hline \multirow[t]{3}{*}{$g_{M N}$} & $g_{\mu \nu}$ & 2 & 848 & $b_{0}$ & $c_{0}$ \\
\hline & $\mathcal{A}_{\mu}$ & 2 & -52 & $b_{1}$ & $c_{0}+c_{1}$ \\
\hline & $\mathcal{A}$ & 1 & 4 & $-b_{1}+b_{3}$ & $-c_{0}-c_{1}+c_{2}+c_{3}$ \\
\hline \multirow[t]{2}{*}{$\psi_{M}$} & $\psi_{\mu}$ & 2 & -233 & $b_{0}+b_{1}$ & $2 c_{0}+c_{1}$ \\
\hline & $\chi$ & 2 & 7 & $b_{2}+b_{3}$ & $c_{1}+2 c_{2}+c_{3}$ \\
\hline \multirow[t]{4}{*}{$A_{M N P}$} & $A_{\mu \nu \rho}$ & 0 & -720 & $b_{0}$ & $c_{0}$ \\
\hline & $A_{\mu \nu}$ & 1 & 364 & $b_{1}$ & $c_{0}+c_{1}$ \\
\hline & $A_{\mu}$ & 2 & -52 & $b_{2}$ & $c_{1}+c_{2}$ \\
\hline & $A$ & 1 & 4 & $b_{3}$ & $c_{2}+c_{3}$ \\
\hline total $f$ & & & & $4\left(b_{0}+b_{1}+b_{2}+b_{3}\right)$ & $4\left(2 c_{0}+2 c_{1}+2 c_{2}+c_{3}\right)$ \\
\hline \multirow[t]{2}{*}{ total $A$} & & & & $-\left(7 b_{0}-5 b_{1}+3 b_{2}-b_{3}\right) / 24$ & $-\left(2 c_{0}-2 c_{1}+2 c_{2}-c_{3}\right) / 24$ \\
\hline & & & & $=-\rho\left(Y^{7}\right) / 24$ & $=-\chi\left(X^{6}\right) / 24$ \\
\hline
\end{tabular}

Table 1. Compactification of $D=11$ supergravity to $D=4$ on manifolds $Y^{7}$ and $X^{6} \times S^{1}$ assuming at least one unbroken supersymmetry. Here, $f(\phi)$ counts the on-shell degrees of freedom and $A(\phi)$ the contribution of a field $\phi$ to the Weyl anomaly coefficient $A$. Note, $b_{k}:=b_{k}\left(Y^{7}\right)$ and $c_{k}:=b_{k}\left(X^{6}\right)$.

(v) Quantum inequivalence of $p$-forms and their duals (section 5): the partition functions for a $p$-form gauge field need not be the same as that of its dual $\tilde{p}=d-p-2[4-6]$. In even dimensions they differ by Euler characteristic terms. In odd dimensions they are equivalent but $\rho$ still makes an appearance via the logarithmic contribution from the non-zero modes of the Laplacian on $p$-forms

$$
(-1)^{p} \rho(X)
$$

It also appears in the partition function of $B F$ theories and the special cases of odd-dimensional Chern-Simons theories. 


\section{The $\rho$-characteristic}

\subsection{Definition}

Let us confine our attention to closed manifolds. In [1-3] it was observed that for a 7manifold $X$, the combination of Betti numbers

$$
\rho(X)=\sum_{p=0}^{3}(-1)^{p}(7-2 p) b_{p}(X)=7 b_{0}-5 b_{1}+3 b_{2}-b_{3},
$$

plays a special role in M-theory compactifications to $D=4$, analogous to that played by Euler characteristic in type II string theory compactifications to $D=4$.

It obviously generalises to arbitrary odd dimensional manifolds $\operatorname{dim} X=2 m+1$,

$$
\rho(X)=\sum_{p=0}^{m}(-1)^{p}(\operatorname{dim} X-2 p) b_{p}(X)
$$

and obeys the Künneth-type formula,

$$
\rho(X \times Y)=\rho(X) \chi(Y)
$$

where $X$ and $Y$ are even and odd dimensional, respectively.

We then define $\rho$ in all dimensions, even and odd, by

$$
\rho(X):=-\sum_{p=0}^{d}(-1)^{p} p b_{p}(X) .
$$

By Poincare duality,

$$
b_{p}(X)=b_{d-p}(X)
$$

this yields (2.2) for $\operatorname{dim} X=2 m+1$ and

$$
\rho(X)=-m \chi(X)
$$

for $\operatorname{dim} X=2 m$.

Obviously, (2.4) is not the unique choice yielding (2.2) in odd dimensions since the Betti numbers are not independent due to Poincaré duality. In particular, we may add any amount of $\chi(X)$ while preserving (2.2). The specific choice (2.4) is natural in many regards and is reminiscent of the Ray-Singer torsion [7]. We are grateful to the referee for drawing our attention to [8] and to a formal similarity between the equations relating $\chi$ and $\rho$ on the one hand and the equations relating the Witten index $\operatorname{Tr}(-1)^{F} e^{-\beta H}$ and the $\mathcal{N}=2, D=2$ Cecotti-Fendley-Intriligator-Vafa (CFIV) index $\operatorname{Tr}(-1)^{F} F e^{-\beta H}$ on the other. Unfortunately, we have been unable to elaborate on this observation. ${ }^{4}$

\footnotetext{
${ }^{4}$ Note, for a supersymmetric sigma model in $D=1,2$ with target space $X$ the CFIV index with its trace restricted to the ground states is identically $\rho(X)$ for the same reason that the Witten index is given by $\chi(X)$. For $\mathcal{N}=(2,2)$ sigma models, $X$ is Kähler and so $\rho(X) \propto \chi(X)$.
} 
The $\rho$-characteristic shares many properties with the Ray-Singer torsion. In particular, using the Künneth formula

$$
b_{r}(X \times Y)=\sum_{p+q=r} b_{p}(X) b_{q}(Y)
$$

we have

$$
\rho(X \times Y)=\rho(X) \chi(Y)+\chi(X) \rho(Y)
$$

for all $X, Y$, which reduces to (2.3) for $X(Y)$ odd (even) dimensional, as required. Note, this same factorisation property is shared by the Ray-Singer and Franz-Reidmeister torsions [7]. Explicitly,

$$
\rho(X \times Y)=\left\{\begin{array}{lll}
-\left(m_{X}+m_{Y}\right) \chi(X) \chi(Y), & \operatorname{dim} X=2 m_{X}, & \operatorname{dim} Y=2 m_{Y} \\
\rho(X) \chi(Y), & X \text { odd, } & Y \text { even } \\
\chi(X) \rho(Y), & X \text { even, } & Y \text { odd } \\
0, & X \text { odd, } & Y \text { odd }
\end{array}\right.
$$

These relations are concisely expressed in terms of the Poincaré polynomials,

$$
P_{X}(z)=\sum_{k}^{d} z^{k} b_{k}(X) .
$$

Recall, the Euler characteristic is given by the Poincaré polynomial evaluated at $z=-1$,

$$
\chi(X)=P_{X}(-1) .
$$

Similarly, (2.4) is simply expressed in terms of the derivative of the Poincaré polynomial,

$$
\rho(X)=P_{X}^{\prime}(-1)
$$

where $P^{\prime}=\partial_{z} P$. Then (2.8) follows immediately from $P_{X \times Y}(z)=P_{X}(z) P_{Y}(z)$,

$$
P_{X \times Y}^{\prime}(-1)=P_{X}^{\prime}(-1) P_{Y}(-1)+P_{X}(-1) P_{Y}^{\prime}(-1) .
$$

Using the freedom to add any amount of $\chi(X)$ to $\rho(X)$ while preserving (2.2) in odd dimensions allows us to introduce a one-parameter family of $\rho_{\lambda}$-characteristics

$$
\rho_{\lambda}(X):=\rho(X)+\lambda \chi(X) .
$$

Two notable members of the family are

$$
\begin{gathered}
\rho_{\left\lfloor\frac{d}{2}\right\rfloor+1}(X)=\sum_{p=0}^{d}(-1)^{p}\left(\left\lfloor\frac{d}{2}\right\rfloor+1-p\right) b_{p}(X) \\
\rho_{\frac{d}{2}}(X)=\sum_{r}^{d}(-1)^{r}\left(\frac{d}{2}-r\right) b_{r}(X) .
\end{gathered}
$$


For $\operatorname{dim} X=2 m$ we have

$$
\begin{gathered}
\rho_{\left\lfloor\frac{d}{2}\right\rfloor+1}(X)=\chi(X) \\
\rho_{\frac{d}{2}}(X)=0 .
\end{gathered}
$$

They also enjoy particularly neat Künneth-type formulae,

$$
\rho_{\left\lfloor\frac{d}{2}\right\rfloor+1}(X \times Y)= \begin{cases}0, & X \text { and } Y \text { odd } \\ \rho_{\left\lfloor\frac{d}{2}\right\rfloor+1}(X) \rho_{\left\lfloor\frac{d}{2}\right\rfloor+1}(Y), & \text { otherwise }\end{cases}
$$

and

$$
\rho_{\frac{d}{2}}(X \times Y)= \begin{cases}0, & X \text { even, } Y \text { even; } \\ \rho(X) \chi(Y), & X \text { odd, } Y \text { even } \\ \chi(X) \rho(Y), & X \text { even, } Y \text { odd } \\ 0, & X \text { odd, } Y \text { odd }\end{cases}
$$

Note, $\rho_{\lambda}$ cannot be defined as a sum of $I_{p}$, the number of $p$-simplices, since the Euler characteristic is the unique (up to a proportionality constant) topological invariant that can be written as a (linear or non-linear) sum of $I_{p}[9,10]$.

\subsection{Uniqueness}

Let us suppose there are topological invariants $\alpha, \beta$ that (i) may be written as a linear combination of Betti numbers and (ii) obeys the Künneth type formula

$$
\alpha(X \times Y)=\beta(X) \chi(Y)
$$

for $X$ odd dimensional and $Y$ even dimensional. We show that $\kappa$ and $\lambda$ are proportional to $\rho$.

Let us first consider the original example of $d=11$ introduced in [1]. Consider 4- and 7-manifolds $X^{4}$ and $X^{7}$ with Betti numbers

$$
a_{p}=b_{p}\left(X^{4} \times X^{7}\right), \quad b_{p}=b_{p}\left(X^{7}\right), \quad c_{p}=b_{p}\left(X^{4}\right)
$$

so that

$$
a_{r}=\sum_{p+q=r} b_{p} c_{q}
$$

Let us suppose $\alpha\left(X^{4} \times X^{7}\right)$ and $\beta\left(X^{7}\right)$ are some linear combination of Betti numbers

$$
\alpha\left(X^{4} \times X^{7}\right)=\sum_{p=0}^{5} A_{p} a_{p}, \quad \beta\left(X^{7}\right)=\sum_{p=0}^{3} B_{p} b_{p} .
$$

If we now demand

$$
\alpha\left(X^{4} \times X^{7}\right)=\beta\left(X^{7}\right) \chi\left(X^{4}\right)
$$


we obtain the multiplication table

$$
\begin{array}{rrrrr} 
& B_{0} b_{0} & B_{1} b_{1} & B_{2} b_{2} & B_{3} b_{3} \\
& & & & \\
2 c_{0} & \left(A_{0}+A_{4}\right) c_{0} b_{0} & \left(A_{1}+A_{5}\right) c_{0} b_{1} & \left(A_{2}+A_{5}\right) c_{0} b_{2} & \left(A_{3}+A_{4}\right) c_{0} b_{3} \\
-2 c_{1} & \left(A_{1}+A_{3}\right) c_{1} b_{0} & \left(A_{2}+A_{4}\right) c_{1} b_{1} & \left(A_{3}+A_{5}\right) c_{1} b_{2} & \left(A_{4}+A_{5}\right) c_{1} b_{3} \\
c_{2} & A_{2} c_{2} b_{0} & A_{3} c_{2} b_{1} & A_{4} c_{2} b_{2} & A_{5} c_{2} b_{3}
\end{array}
$$

where we have used (2.21) and Poincaré duality to express all Betti numbers in terms of $c_{p}$ and $b_{q}$ for $p<3, q<4$. We use the overall scaling to fix $A_{0}=11$. This implies the system of 12 equations,

$$
\begin{aligned}
& 2 B_{0}=11+A_{4}=-A_{1}-A_{3}=2 A_{2} \\
& 2 B_{1}=A_{1}+A_{5}=-A_{2}-A_{4}=2 A_{3} \\
& 2 B_{2}=A_{2}+A_{5}=-A_{3}-A_{5}=2 A_{4} \\
& 2 B_{3}=A_{3}+A_{4}=-A_{4}-A_{5}=2 A_{5}
\end{aligned}
$$

nine of which are independent, which (e.g. sequentially eliminating $A_{5}, A_{4} \ldots$ first) immediately reduce to

$$
A_{p}=(-)^{p}(11-2 p), \quad B_{p}=(-)^{p}(7-2 p) .
$$

Hence,

$$
\alpha\left(X^{4} \times X^{7}\right)=\rho\left(X^{4} \times X^{7}\right), \quad \beta\left(X^{7}\right)=\rho\left(X^{7}\right)
$$

Thus not only does $\rho$ satisfy the Künneth-type formula, it is the unique linear combination of Betti numbers to do so.

This immediately generalises to arbitrary $X^{d-d^{\prime}}, X^{d^{\prime}}$, assuming with out loss of generality $d^{\prime}>d / 2$. Let $d=2 n+1$ and $d^{\prime}=2 m+1$. Then we have a set of $n+m+1$ independent equations (again fixing $A_{0}=d$ ) yielding

$$
A_{p}=(-)^{p}(d-2 p), \quad B_{p}=(-)^{p}\left(d^{\prime}-2 p\right) .
$$

for the $n+m+2$ coefficients $A_{p}, B_{q}, p=0, \ldots n, q=0, \ldots m$.

\subsection{Cosets}

Consider a compact connected Lie group $G$ of rank $r$. Its Poincaré polynomial is given by

$$
P_{G}(z)=\prod_{i=1}^{r}\left(1+z^{g_{i}}\right)
$$

where $g_{i}=2 c_{i}-1$, with $c_{i}$ the degree of the $i^{\text {th }}$ Casimir invariant of $G$.

Evidently, the Euler characteristic vanishes

$$
\chi(G)=P_{G}(-1)=0 .
$$


The same is true for $\rho$ if $r>1$,

$$
\rho(G)=P_{G}^{\prime}(-1)
$$

while

$$
\rho(\mathrm{U}(1))=1, \quad \rho(\mathrm{SU}(2))=3 .
$$

For cosets on the other hand, both $\chi$ and $\rho$ can be non-trivial. Consider a compact connected Lie group $G$ of rank $r$ and a compact connected Lie subgroup $H$ of rank $s \leq r$. Let us denote their Poincaré polynomials by

$$
P_{G}(z)=\prod_{i=1}^{r}\left(1+z^{g_{i}}\right), \quad P_{H}(z)=\prod_{i=1}^{s}\left(1+z^{h_{i}}\right)
$$

where $g_{i}=2 c(G)_{i}-1, h_{i}=2 c(H)_{i}-1$, with $c(G)_{i}$ and $c(H)_{i}$ the degree of the $i^{t h}$ Casimir invariant of $G$ and $H$, respectively. Then the Poincaré polynomial of the coset manifold is

$$
P_{G / H}(z)=\frac{\prod_{i=1}^{s}\left(1-z^{g_{i}+1}\right) \prod_{i=s+1}^{r}\left(1+z^{g_{i}}\right)}{\prod_{i=1}^{s}\left(1-z^{h_{i}+1}\right)},
$$

where

$$
P_{\widehat{G / K}}(z)=\sum_{i=s}^{r} z^{g_{i}}
$$

is the Poincaré polynomial of the Samelson subspace for the pair $(G, H)$. See, for example, [11].

From (2.34) we have

$$
\begin{aligned}
\rho(G / H)=( & -\frac{\sum_{k=1}^{s}\left(1-z^{g_{1}+1}\right) \cdots\left(g_{k}+1\right) z^{g_{k}} \cdots\left(1-z^{g_{s}+1}\right) \prod_{i=s+1}^{r}\left(1+z^{g_{i}}\right)}{\prod_{i=1}^{s}\left(1-z^{h_{i}+1}\right)} \\
& +\frac{\prod_{i=1}^{s}\left(1-z^{g_{i}+1}\right) \sum_{k=s+1}^{r}\left(1+z^{g_{s+1}}\right) \ldots g_{k} z^{g_{k}-1} \cdots\left(1+z^{g_{r}}\right)}{\prod_{i=1}^{s}\left(1-z^{h_{i}+1}\right)} \\
& \left.+\sum_{k=1}^{s} \frac{\prod_{i=1}^{s}\left(1-z^{g_{i}+1}\right) \prod_{i=s+1}^{r}\left(1+z^{g_{i}}\right)\left(h_{k}+1\right) z^{h_{k}}}{\left(1-z^{h_{1}+1}\right) \cdots\left(1-z^{h_{k}+1}\right)^{2} \cdots\left(1-z^{h_{s}+1}\right)}\right)\left.\right|_{z=-1}
\end{aligned}
$$

This goes as $\lim _{x \rightarrow 0} x^{r-(s+1)}$ and, hence, $\rho$ can be non-zero only if $r=s$ or $r=s+1$.

For $r=s$ we have

$$
\frac{2}{\operatorname{dim} G / H} \rho(G / H)=\chi(G / H)=-\prod_{i=1}^{r} \frac{g_{i}+1}{h_{i}+1},
$$

whereas for $r=s+1$ we get

$$
\rho(G / H)=g_{s+1} \prod_{i=1}^{s} \frac{g_{i}+1}{h_{i}+1}
$$

A number of examples are given in table 2 . 


\begin{tabular}{|c|c|c|c|c|c|}
\hline$M$ & $G$ & $H$ & $P_{M}(z)$ & $\chi(M)$ & $\rho(M)$ \\
\hline$S^{2 n}$ & $\mathrm{SO}(2 n+1)$ & $\mathrm{SO}(2 n)$ & $1+z^{2 n}$ & 2 & $-2 n$ \\
$S^{2 n+1}$ & $\mathrm{SO}(2 n+2)$ & $\mathrm{SO}(2 n+1)$ & $1+z^{2 n+1}$ & 0 & $2 n+1$ \\
$\mathrm{CP}^{n}$ & $\mathrm{SU}(n+1)$ & $\mathrm{U}(n)$ & $\sum_{k=0}^{n} z^{2 k}$ & $n+1$ & $-n(n+1)$ \\
$G / H$ & $\mathrm{SU}(4)$ & $\mathrm{SU}(2) \times \mathrm{SU}(2)$ & $1+z^{4}+z^{5}+z^{9}$ & 0 & 10 \\
\hline
\end{tabular}

Table 2. The $\chi$ - and $\rho$-characteristics for various coset spaces $M=G / H$.

Since $G$ can be regarded as a bundle $H \rightarrow G \rightarrow G / H$ we have $\chi(G)=\chi(H) \chi(G / H)$, which is trivially satisfied since $\chi(G)=0$ for all $G$. Nonetheless, although $\rho(G)=0$ for all $G$ with $r>1$, we can formally define, for $G$ and $H$ of equal rank

$$
\frac{\rho(G)}{\rho(H)}:=\lim _{z \rightarrow-1} \frac{P_{G}^{\prime}(z)}{P_{H}^{\prime}(z)}=\prod_{i=1}^{r} \frac{g_{i}}{h_{i}} .
$$

Then

$$
\left.\frac{\rho(G)}{\rho(H)}\right|_{g_{i} \mapsto g_{i}+1, h_{i} \mapsto h_{i}+1}=-\chi(G / H),
$$

resembling a Künneth-type formula.

\section{$3 \quad$ Mirror symmetry}

\subsection{Conventional mirror symmetry: Calabi-Yau and Joyce}

Consider Calabi-Yau manifolds $X$ of complex dimension $n$. Denoting the Hodge numbers by $h^{p, q}=h^{n-p, n-q}$, the Betti numbers are

$$
b_{k}=\sum_{p+q=k} h^{p, q}
$$

and the Euler characteristic is

$$
\chi(n):=\chi(X)=\sum_{k=0}^{k=n}(-1)^{k} b_{k}=\sum_{k=0}^{k=n}(-1)^{k} \sum_{p+q=k} h^{p, q} .
$$

Their mirrors $\check{X}$ are defined by

$$
\check{h}^{p, q}=h^{n-p, q},
$$

where $\check{h}^{p, q}:=h^{p, q}(\check{X})$.

Then for odd complex dimension $n=2 r+1$,

$$
\check{\chi}(2 r+1)=-\chi(2 r+1),
$$


where $\check{\chi}(n):=\chi(\check{X})$ and

$$
\begin{aligned}
& \text { IIA on } X \equiv \text { IIB on } \check{X} \\
& \text { IIB on } X \equiv \text { IIA on } \check{X}
\end{aligned}
$$

whereas for even complex dimension $n=2 r$

$$
\check{\chi}(2 r)=\chi(2 r)
$$

and

$$
\begin{aligned}
& \text { IIA on } X \equiv \text { IIA on } \check{X} \\
& \text { IIB on } X \equiv \text { IIB on } \check{X}
\end{aligned}
$$

Our focus will be on the Betti, rather than the Hodge, numbers. It is therefore illustrative to rephrase the CY mirror symmetry in terms Betti numbers. The CY3 Hodge numbers are

$$
h^{p, q}=\left(\begin{array}{cccc}
1 & 0 & 0 & 1 \\
0 & a & a & 0 \\
0 & \check{a} & a & 0 \\
1 & 0 & 0 & 1
\end{array}\right)
$$

where $a=h^{1,1}$ and $\check{a}=h^{2,1}$ are the two independent entries. Written this way,

$$
\left(b_{0}, b_{1}, b_{2}, b_{3}\right)=(1,0, a, 2+2 \check{a})
$$

and

$$
\chi(3)=2(a-\check{a}) .
$$

The mirror transformation is then

$$
a \leftrightarrow \check{a}
$$

which manifestly implies $\check{\chi}(3)=-\chi(3)$. In terms of the Betti numbers we have

$$
\Delta b_{2}=\check{a}-a=-\chi(3) / 2, \quad \Delta b_{3}=(2+2 a)-(2+2 \check{a})=\chi(3)
$$

as given in table 3 .

Similarly, for CY5 we have

$$
h^{p, q}=\left(\begin{array}{cccccc}
1 & 0 & 0 & 0 & 0 & 1 \\
0 & a & b & \breve{b} & \check{a} & 0 \\
0 & b & c & \check{c} & \check{b} & 0 \\
0 & \breve{b} & \check{c} & c & b & 0 \\
0 & \check{a} & \breve{b} & b & a & 0 \\
1 & 0 & 0 & 0 & 0 & 1
\end{array}\right)
$$

so that

$$
\left(b_{0}, b_{1}, b_{2}, b_{3}, b_{4}, b_{5}\right)=(1,0, a, 2 b, 2 \check{b}+c, 2+2 \check{a}+2 \check{c})
$$


and

$$
\chi(5)=2(a-\check{a})-4(b-\check{b})+2(c-\check{c}) .
$$

The mirror transformation is then

$$
a \leftrightarrow \check{a}, \quad b \leftrightarrow \check{b}, \quad c \leftrightarrow \check{c}
$$

which manifestly implies $\check{\chi}(5)=-\chi(5)$. Letting

$$
A=a-\check{a}, \quad B=b-\check{b}, \quad C=c-\check{c}
$$

in terms of the Betti numbers we have

$$
\Delta b_{2}=-A, \quad \Delta b_{3}=-2 B, \quad \Delta b_{4}=2 B-C, \quad \Delta b_{5}=2 A+2 C
$$

as given in table 3 .

The extensions of the mirror map for Calabi-Yau manifolds [12-16], with holonomy $\mathrm{SU}(n)$, to mirror maps for $d=7$ Joyce manifolds, with holonomy $G_{2}$, and $d=8 \operatorname{Spin}(7)$ manifolds were considered in [17-20]. See also [21] for a recent treatment of mirror symmetry for generalised connected sum constructions of $G_{2}$ and $\operatorname{Spin}(7)$ manifolds. In these cases $b_{1}=0$. A further extension to more general manifolds, both even and odd was considered in [1] without the condition that $b_{1}=0$.

A necessary condition for the quantum equivalence of type IIA/B string theories compactified on mirror exceptional holonomy manifolds is that the dimension of the space of exactly marginal operators on the worldsheet is preserved [17]. This is given by the number of moduli in NS-NS sector. Since the metric is determined by the associative 3 -form for torsionless $G_{2}$ manifolds $X$, the number of geometric moduli is given $b_{3}(X)[19,20]$, so the number of NS-NS moduli is given by

$$
b_{2}(X)+b_{3}(X)
$$

Similarly, for $\operatorname{Spin}(7)$ manifolds $X$, the number of geometric moduli is given $b_{4}^{-}(X)+$ $1[19,20]$, so the number of NS-NS moduli is given by

$$
b_{2}(X)+b_{4}^{-}(X)+1 \text {. }
$$

In all cases, a necessary condition for quantum equivalence is that the number of NS-NS moduli are preserved. Since $b_{1}=0$ is also preserved for supersymmetry, this implies that the NS-NS sector is left invariant.

\subsection{Generalised mirror maps; $\chi \rightarrow \pm \chi$ in even $d$}

The generalised mirror transformations are not peculiar to string theory or even supersymmetry, but given the preceding discussion we will nevertheless maintain the property of leaving invariant the NS sectors and preserving/interchanging the RR sectors of Type IIA and IIB. Note, these conditions are necessary but possibly not sufficient for quantum equivalence of type IIA/B compactified on mirror manifolds, hence the use of mirror maps 
as opposed to mirror symmetry. The known mirror symmetries are all examples of the generalised mirror maps considered here.

Let $\chi_{\mathrm{e}}(Y)$ be the sum of the even Betti numbers and $\chi_{\mathrm{o}}(Y)$ the odd Betti numbers of the compactifying manifold $Y$,

$$
\chi_{\mathrm{e}}(Y)=\sum_{k=0} b_{2 k}(Y), \quad \chi_{\mathrm{o}}(Y)=\sum_{k=0} b_{2 k+1}(Y),
$$

so that

$$
\chi(Y)=\chi_{\mathrm{e}}(Y)-\chi_{\mathrm{o}}(Y)
$$

To calculate the number of degrees of freedom, we note that for $\operatorname{dim} Y \leq 7$ preserving some supersymmetry,

$$
f(\mathrm{NS}-\mathrm{NS})=f(\mathrm{NS}-\mathrm{R})=f(\mathrm{R}-\mathrm{NS})=f(\mathrm{RR})=f / 4
$$

for both Type IIA and IIB, where $f(S)$ denotes the degrees of freedom of the sector $S$. Moreover, from table 4 , using that a $p$-form in $D$ dimensions carries $(D-2) ! /(D-2-p) ! p$ ! degrees of freedom, we find

$$
f(\mathrm{RR})=2^{(6-d)}\left(\chi_{\mathrm{e}}(Y)+\chi_{\mathrm{o}}(Y)\right)
$$

and hence

$$
2^{(d-8)} f=\chi_{\mathrm{e}}(Y)+\chi_{\mathrm{o}}(Y)
$$

for both Type IIA and Type IIB. This breaks down for $d=8$, where instead

$$
\begin{aligned}
& f_{\mathrm{IIA}}(\mathrm{RR})=\frac{1}{2} \chi_{\mathrm{o}}(Y) \\
& f_{\mathrm{IIB}}(\mathrm{RR})=\frac{1}{2} \chi_{\mathrm{e}}(Y)
\end{aligned}
$$

so that for all $d \leq 8$ we have the weaker statement

$$
f_{\mathrm{IIA}}(\mathrm{RR})+f_{\mathrm{IIB}}(\mathrm{RR})=2^{(7-d)}\left(\chi_{\mathrm{e}}(Y)+\chi_{\mathrm{o}}(Y)\right) .
$$

For the generalized mirror symmetry we seek a transformation of the Betti numbers $\Delta b_{n}$ in $d=2 m$ that preserves the number of degrees of freedom $\Delta f=0$ and for which $\Delta \chi=-2 \chi, m$ odd, and $\Delta \chi=0, m$ even. Taking into account (3.23), (3.24) and (3.26), this is easily achieved by demanding

$$
\chi_{\mathrm{e} / \mathrm{o}}(Y) \mapsto \begin{cases}\chi_{\mathrm{o} / \mathrm{e}}(Y) m & \text { odd } \\ \chi_{\mathrm{e} / \mathrm{o}}(Y) m & \text { even. }\end{cases}
$$

But $b_{0}=b_{d}$ is always unity and we do not want to change the number of supersymmetries. For $d<8$ this implies $\Delta b_{1}=0$, since $b_{1}$ and the degree of supersymmetry are bijective. This follows from the fact that any compact connected Ricci-flat manifold with $\operatorname{dim} X=n$ is of the form $X=\left(T^{k} \times X^{n-k}\right) / \Gamma$, where $\Gamma$ is a freely acting finite subgroup of the isometries of $X^{n-k}$, where $X^{n-k}$ is simply connected and $b_{1}\left(X^{n}\right)=k$ [22]. For $d=8$ the relation 


\begin{tabular}{|l|c|c|c|c|c|c|}
\hline$d$ & $\Delta b_{2}$ & $\Delta b_{3}$ & $\Delta b_{4}$ & $\Delta b_{5}$ & $\Delta \chi$ & $\Delta \rho$ \\
\hline \multirow{6}{*}{6} & $-\chi / 2$ & $\chi$ & & & & \\
7 & $-\rho / 2$ & $\rho / 2$ & & & $-2 \chi$ & $-2 \rho$ \\
8 & $n$ & 0 & $-2 n$ & & 0 & $-2 \rho$ \\
9 & $n$ & $n$ & $-2 n$ & & 0 & 0 \\
10 & $-\chi / 2-2 B+C$ & $-2 B$ & $2 B-C$ & $\chi+4 B$ & $-2 \chi$ & $-2 \rho$ \\
11 & $-\rho / 2-2 B+C$ & $-\rho / 2-4 B+C$ & $-C$ & $\rho+6 B-C$ & 0 & $-2 \rho$ \\
\hline
\end{tabular}

Table 3. Generalised mirror maps and their corresponding $\Delta \chi, \Delta \rho$. By Poincaré duality $\Delta b_{k}=$ $\Delta b_{d-k}$. Here, $n, A, B, C$ are arbitrary integers satisfying $\chi=2 A-4 B+2 C$ and $\rho=2 A-4 B+2 C$, consistent with the special case of Calabi-Yau fivefolds, cf. (3.15) and (3.18). Note, we demand $\Delta b_{d / 2}^{+}=\Delta b_{d / 2}^{-}$consistent with the requirement that the NS-NS sector is left invariant for CalabiYau and $\operatorname{Spin}(7)$ compactifications.

between $b_{1}$ and the number of supercharges breaks down. For example, $T^{1} \times X$ and $Y$, for $X$ a $G_{2}$-manifold and $Y$ a Calabi-Yau fourfold, both preserve 1/8 supersymmetry but have distinct $b_{1}$. However, we shall none the less insist on $\Delta b_{1}=0$ in view of the conventional mirror symmetry of Calabi-Yau manifolds.

Hence, we require

$$
\begin{aligned}
\sum_{n=2}^{d-2} \Delta b_{n} & =0 \\
\sum_{n=2}^{d-2}(-1)^{n} \Delta b_{n} & =\left\{\begin{array}{lll}
-2 \chi & m & \text { odd } \\
0 & m & \text { even }
\end{array}\right.
\end{aligned}
$$

and $d=6$ is the lowest dimension for which this mirror transformation is non-trivial. There are non-trivial mirror symmetries for $d<6$, for example $T$-duality or mirror symmetries taking $K 3$ surfaces into themselves [23], but they act trivially on the Betti numbers so are not captured by the mirror maps considered here.

The $d=(6,8,10)$ transformations are shown in table 3 . Note, for $d=6$ only $\Delta b_{2}, \Delta b_{3}$ are non-trivial so there are no free parameters in the mirror map. In $d=8,10$ there are one and two arbitrary integer parameters, respectively, defining a family of mirror maps. Further requirements may restrict these. For instance, in the case of Calabi-Yau manifolds the mirror symmetry (as opposed to map) is given by the finer Hodge number transformation (3.3) by the requirement of quantum equivalence of type II string theories compactified on the mirror manifolds.

The degree of freedom invariance under the mirror maps (3.28) can be made explicit by defining a formal Euler characteristic of R-R field strengths. Since the NS-NS sector is invariant, we focus on the bosonic R-R field strength content of Type IIA (even forms $F_{0}, F_{2}, F_{4}$ ) and Type IIB (odd forms $F_{1}, F_{3}, F_{5}^{-}$). Performing a Kaluza-Klein reduction on 


$$
\begin{aligned}
& F_{0}(X \times Y)=F_{0}(X) b_{0}(Y) \\
& F_{1}(X \times Y)=F_{0}(X) b_{1}(Y)+F_{1}(X) b_{0}(Y) \\
& F_{2}(X \times Y)=F_{0}(X) b_{2}(Y)+F_{1}(X) b_{1}(Y)+F_{2}(X) b_{0}(Y) \\
& F_{3}(X \times Y)=F_{0}(X) b_{3}(Y)+F_{1}(X) b_{2}(Y)+F_{2}(X) b_{1}(Y)+F_{3}(X) b_{0}(Y) \\
& F_{4}(X \times Y)=F_{0}(X) b_{4}(Y)+F_{1}(X) b_{3}(Y)+F_{2}(X) b_{2}(Y)+F_{3}(X) b_{1}(Y)+F_{4}(X) b_{0}(Y) \\
& F_{5}^{-}(X \times Y)=F_{0}(X) b_{5}(Y)+F_{1}(X) b_{4}(Y)+\cdots F_{m}^{+}(X) b_{(5-m)}^{-}(Y)+F_{m}^{-}(X) b_{(5-m)}^{+}(Y) \ldots+F_{5}(X) b_{0}(Y) \\
& b_{0}(X \times Y)=b_{0}(X) b_{0}(Y) \\
& b_{1}(X \times Y)=b_{0}(X) b_{1}(Y)+b_{1}(X) b_{0}(Y) \\
& b_{2}(X \times Y)=b_{0}(X) b_{2}(Y)+b_{1}(X) b_{1}(Y)+b_{2}(X) b_{0}(Y) \\
& b_{3}(X \times Y)=b_{0}(X) b_{3}(Y)+b_{1}(X) b_{2}(Y)+b_{2}(X) b_{1}(Y)+b_{3}(X) b_{0}(Y)+ \\
& b_{4}(X \times Y)=b_{0}(X) b_{4}(Y)+b_{1}(X) b_{3}(Y)+b_{2}(X) b_{2}(Y)+b_{3}(X) b_{1}(Y)+b_{4}(X) b_{0}(Y) \\
& b_{5}^{-}(X \times Y)=b_{0}(X) b_{5}(Y)+b_{1}(X) b_{4}(Y)+\cdots b_{m}^{+}(X) b_{(5-m)}^{-}(Y)+b_{m}^{-}(X) b_{(5-m)}^{+}(Y)+\ldots+b_{5}(X) b_{0}(Y)
\end{aligned}
$$

Table 4. Comparison of $F_{p}$ content of compactified IIA and IIB and the Künneth formula. The notation $F_{p}(X) b_{q}(Y)$ should read as there are $p$-form field strengths on $X$ with multiplicity $b_{q}(Y)$.

$M=X \times Y$, where $Y$ is a $d$-dimensional Riemannian and $X$ is a $(D=10-d)$-dimensional Lorentzian manifold, yields the $F$ content shown in table 4 .

Motivated by table 4 let us introduce the formal $p$-form field strength "Euler characteristic"

$$
\chi(X)=F_{0}(X)-F_{1}(X)+F_{2}(X)+\cdots
$$

and "Hirzebruch signature"

$$
\tau\left(X^{\prime}\right)=F_{m}^{+}(X)-F_{m}^{-}(X) m \text { even }
$$

akin to

$$
\chi(Y)=b_{0}(Y)-b_{1}(Y)+b_{2}(Y)+\cdots
$$

and

$$
\tau(Y)=b_{m}^{+}(Y)-b_{m}^{-}(Y) m \text { odd. }
$$

Note $X$ has Lorentzian spacetime signature and $\tau(X)$ is defined in $2 \bmod 4$ while $Y$ has Euclidean signature and $\tau(Y)$ is defined in $0 \bmod 4$.

If we define

$$
\Sigma_{\alpha}^{ \pm}(M)=\frac{1}{2}\left[\chi_{\alpha}(M) \pm \tau_{\alpha}(M)\right], \quad \alpha=\mathrm{e}, \mathrm{o}
$$

then field content of the R-R sector can be summarised by

$$
\begin{aligned}
& \mathrm{IIA}=\Sigma_{\mathrm{e}}^{+}(X) \Sigma_{\mathrm{e}}^{-}(Y)+\Sigma_{\mathrm{e}}^{-}(X) \Sigma_{\mathrm{e}}^{+}(Y)+\Sigma_{\mathrm{o}}^{+}(X) \Sigma_{\mathrm{o}}^{-}(Y)+\Sigma_{\mathrm{o}}^{-}(X) \Sigma_{\mathrm{o}}^{+}(Y) \\
& \mathrm{IIB}=\Sigma_{\mathrm{e}}^{+}(X) \Sigma_{\mathrm{o}}^{-}(Y)+\Sigma_{\mathrm{e}}^{-}(X) \Sigma_{\mathrm{o}}^{+}(Y)+\Sigma_{\mathrm{o}}^{+}(X) \Sigma_{\mathrm{e}}^{-}(Y)+\Sigma_{\mathrm{o}}^{-}(X) \Sigma_{\mathrm{e}}^{+}(Y)
\end{aligned}
$$

and

$$
\begin{aligned}
\mathrm{IIA}-\mathrm{IIB} & =[\chi(X)+\tau(X)][\chi(Y)-\tau(Y)]+[\chi(X)-\tau(X)][\chi(Y)+\tau(Y)] \\
& =\chi(X) \chi(Y)+\tau(X) \tau(Y)=\chi(X \times Y)-\tau(X \times Y)
\end{aligned}
$$




\begin{tabular}{|r|c|c|}
\hline$d$ & Type IIA & Type IIB \\
\hline 0 & $\left(F_{0}+F_{2}+F_{4}\right) b_{0}$ & $\left(F_{1}+F_{3}+F_{5}{ }^{-}\right) b_{0}$ \\
1 & $\left(F_{0}+F_{1}+F_{2}+F_{3}+F_{4}\right) b_{0}$ & $\left(F_{0}+F_{1}+F_{2}+F_{3}+F_{4}\right) b_{0}$ \\
2 & $\left(2 F_{0}+2 F_{2}+F_{4}\right) b_{0}+\left(F_{1}+F_{3}\right) b_{1}$ & $2\left(F_{1}+F_{3}\right) b_{0}+\left(F_{0}+F_{2}+F_{4} / 2\right) b_{1} / 2+\left(F_{0}+F_{2}+F_{4} / 2\right) b_{1} / 2$ \\
3 & $\left(F_{0}+F_{1}+F_{2}+F_{3}\right)\left(b_{0}+b_{1}\right)$ & $\left(F_{0}+F_{1}+F_{2}+F_{3}\right)\left(b_{0}+b_{1}\right)$ \\
4 & $\left(F_{0}+F_{2}\right)\left(2 b_{0}+b_{2}\right)+\left(2 F_{1}+F_{3}\right) b_{1}$ & $\left(F_{1}+F_{3}{ }^{-}\right)\left(b_{0}+b_{2}{ }^{+}\right)+\left(F_{1}+F_{3}{ }^{+}\right)\left(b_{0}+b_{2}{ }^{-}\right)+2\left(F_{0}+F_{2}\right) b_{1}$ \\
5 & $\left(b_{0}+b_{1}+b_{2}\right)\left(F_{0}+F_{1}+F_{2}\right)$ & $\left(b_{0}+b_{1}+b_{2}\right)\left(F_{0}+F_{1}+F_{2}\right)$ \\
6 & $\left(b_{0}+b_{2}\right)\left(2 F_{0}+F_{2}\right)+\left(2 b_{1}+b_{3}\right) F_{1}$ & $\left(b_{1}+b_{3} / 2\right)\left(F_{0}+F_{2} / 2\right)+\left(b_{1}+b_{3} / 2\right)\left(F_{0}+F_{2} / 2\right)+2\left(b_{0}+b_{2}\right) F_{1}$ \\
7 & $\left(b_{0}+b_{1}+b_{2}+b_{3}\right)\left(F_{0}+F_{1}\right)$ & $\left(b_{0}+b_{1}+b_{2}+b_{3}\right)\left(F_{0}+F_{1}\right)$ \\
8 & $\left(2 b_{0}+2 b_{2}+b_{4}\right) F_{0}+\left(b_{1}+b_{3}\right) F_{1}$ & $2\left(b_{1}+b_{3}\right) F_{0}+\left(b_{0}+b_{2}+b_{4}{ }^{+}\right) F_{1}^{-}+\left(b_{0}+b_{2}+b_{4}{ }^{-}\right) F_{1}+$ \\
9 & $\left(b_{0}+b_{1}+b_{2}+b_{3}+b_{4}\right) F_{0}$ & $\left(b_{0}+b_{1}+b_{2}+b_{3}+b_{4}\right) F_{0}$ \\
10 & $\left(b_{0}+b_{2}+b_{4}\right) F_{0}$ & $\left(b_{1}+b_{3}+b_{5}{ }^{-}\right) F_{0}$ \\
\hline
\end{tabular}

Table 5. Betti/flux (b/F) duality on $X^{(10-d)} \times Y^{d}$.

The virtue of this formalism is that invariance under the generalized mirror symmetry (3.28) is manifest

$$
\begin{aligned}
& \mathrm{IIA} \rightarrow \text { IIB IIB } \rightarrow \text { IIA } m \text { odd; } \\
& \mathrm{IIA} \rightarrow \text { IIA IIB } \rightarrow \text { IIB } m \text { even. }
\end{aligned}
$$

for $\operatorname{dim} Y=2 m$.

However this formalism also reveals an unexpected symmetry, interesting in its own right, namely interchanging the Betti numbers and the fluxes while simultaneously interchanging the spacetime and compact manifolds. See table 3.2.

Note, in deriving these results, we have assumed Poincaré duality for manifold $Y$,

$$
b_{p}(Y)=b_{d-p}(Y)
$$

and analogously we have freely dualised

$$
F_{p}(X) \leftrightarrow F_{D-p}(X)
$$

which is superficially possible in the R-R sector. As we shall see in section 4 , a $p$-form and its dual can have different Weyl anomalies. Moreover in 5.2.1 we shall see in greater detail how their partition functions may differ. However, since $Y$ is assumed to be Ricci flat and to preserve at least one supersymmetry, $X$ is a Minkowski spacetime and no duality anomaly can arise.

Even if this were not the case, one could adopt the democratic formulation [24] whereby in $D=10$ spacetime dimensions IIA includes not only $\left(F_{0}, F_{2}, F_{4}\right)$ but also their duals $\left(F_{10}, F_{8}, F_{6}\right)$ and IIB includes not only $\left(F_{1}, F_{3}, F_{5}{ }^{+}\right)$but also $\left(F_{9}, F_{7}, F_{5}^{-}\right)$. Moreover, 
recognising that the anomalies given by the Seeley-deWitt coefficient vanish in odd dimensions and therefore after compactification to an even dimension the massive and massless contributions to the anomaly must cancel $[25,26]$, as we demonstrate in section 5 . The assumption that IIA is duality anomaly free once its massive states are included is motivated by its M-theory uplift, where there can be no anomaly. Given the equivalence between type IIA and II B on a circle, including the massive states in type IIB one can apply the same logic.

\subsection{Generalised mirror maps; $\rho \rightarrow \pm \rho$ in odd $d$}

Following the treatment of $\chi$ in even dimensions we let

$$
\rho_{\mathrm{e}}(Y)=-\sum_{k=0} 2 k b_{2 k}(Y), \quad \rho_{\mathrm{o}}(Y)=-\sum_{k=0}(2 k+1) b_{2 k+1}(Y),
$$

so that

$$
\rho(Y)=\rho_{\mathrm{e}}(Y)-\rho_{\mathrm{o}}(Y)
$$

and the degrees of freedom $f$ for $d \leq 7$ are given by

$$
2^{(d-8)} d f=\rho_{\mathrm{e}}(Y)+\rho_{\mathrm{o}}(Y) .
$$

For the generalised mirror symmetry we seek a transformation of the Betti numbers $\Delta b_{n}$ in $d=2 m+1$ that preserves the number of degrees of freedom $\Delta f=0$ and for which $\Delta \rho=-2 \rho, \mathrm{m}$ odd, and $\Delta \rho=0, m$ even. This is easily achieved by

$$
\rho_{\mathrm{e} / \mathrm{o}}(Y) \mapsto \begin{cases}\rho_{\mathrm{o} / \mathrm{e}}(Y) m & \text { odd; } \\ \rho_{\mathrm{e} / \mathrm{o}}(Y) m & \text { even. }\end{cases}
$$

Once again $b_{0}$ is always unity and we do not want to change the number of supersymmetries so cannot change $b_{1}$ in $d<8$ (and we apply this restriction for $d \geq 8$ ). Hence

$$
\begin{aligned}
\sum_{n=2}^{d-2} \Delta b_{n} & =0 \\
\sum_{n=2}^{d-2}(-1)^{n} n \Delta b_{n} & =\left\{\begin{array}{lll}
-2 \rho & m \text { odd } \\
0 & m & \text { even }
\end{array}\right.
\end{aligned}
$$

and $d=7$ is the lowest dimension for which this mirror transformation is defined. The $d=(7,9,11)$ transformations are shown in table 3 . As a consistency check we note that for $d=7$ the condition (3.46) reduces to

$$
\Delta b_{2}+\Delta b_{3}=0
$$

as found in $[17,19,20]$ for $G_{2}$-manifolds. Note, $b_{2}+b_{3}$ is also the number of spin- $1 / 2$ fermions, cf. table 1 , and since we are preserving the degree of supersymmetry, this implies that (3.48) is also required by the invariance of the degrees of freedom. Examples for $G_{2}$-manifolds may be found in table 6 . 


\begin{tabular}{|c|c|c|c|c|}
\hline$\left(b_{0}, b_{1}, b_{2}, b_{3}\right)$ & $k$ & $\left(\Delta b_{2}, \Delta b_{3}\right)$ & $\rho$ & $f$ \\
\hline$(1,0,8,47)$ & 0 & $(8,-8)$ & -16 & 224 \\
$(1,0,0,31+2 k)$ & $0, \ldots, 22,24,29,30$ & $(12+k,-12-k)$ & $-24-2 k$ & $128+8 k$ \\
$(1,0,1,30+2 k)$ & $0, \ldots, 19,21$ & $(10+k,-10-k)$ & $-20-2 k$ & $128+8 k$ \\
$(1,0,2,29+2 k)$ & $0, \ldots, 10,12,13,15$ & $(8+k,-8-k)$ & $-16-2 k$ & $128+8 k$ \\
$(1,0,3,28+2 k)$ & $0, \ldots, 7,9,10$ & $(6+k,-6-k)$ & $-12-2 k$ & $128+8 k$ \\
$(1,0,4,27+2 k)$ & $0,1,2,3$ & $(4+k,-4-k)$ & $-8-2 k$ & $128+8 k$ \\
$(1,0,5,26)$ & 0 & $(2,-2)$ & -4 & 128 \\
\hline
\end{tabular}

Table 6. Generalised mirror map for examples of $G_{2}$ manifolds.

\section{Weyl anomalies}

\subsection{Weyl anomalies}

Weyl anomalies [27] take their most pristine form in the context of conformal field theories in a background gravitational field, for which the trace of the stress tensor is classically zero but nonzero at the quantum level (e.g. conformal scalars and massless fermions in any $D$, Maxwell/Yang-Mills in $D=4, p$-form gauge fields in $D=2 p+2$, Conformal Supergravity in $D=(2,4,6)$ ). For other theories (e.g. Maxwell/Yang-Mills for $D \neq 4$, pure quantum gravity for $D>2$, or any theory with mass terms) the "anomalies" will still survive, but will be accompanied by contributions from $\left\langle g^{\alpha \beta} T_{\alpha \beta}\right\rangle$ expected anyway through the lack of conformal invariance. Since the anomaly arises because the operations of regularizing and taking the trace do not commute, the anomaly $\mathcal{A}$ in a theory which is not classically Weyl invariant may be defined as [28-30]:

$$
\mathcal{A}^{\mathrm{W}}:=g^{\alpha \beta}\left\langle T_{\alpha \beta}\right\rangle_{\mathrm{reg}}-\left\langle g^{\alpha \beta} T_{\alpha \beta}\right\rangle_{\mathrm{reg}} .
$$

Of course, the second term happens to vanish when the classical invariance is present. $\mathcal{A}^{\mathrm{W}}$ is given by the Seeley-deWitt coefficient $B_{d}$ and will be local, which $g^{\alpha \beta}\left\langle T_{\alpha \beta}\right\rangle_{\text {reg }}$ in general is not. That it still makes sense to talk of an anomaly in the absence of a symmetry is also familiar from the divergence of the axial vector current $[31,32]$ where the operations of regularizing and taking the divergence do not commute

$$
\mathcal{A}^{\text {Axial }}:=\partial_{\mu}\left\langle\left(\sqrt{g} J^{\mu}\right)\right\rangle_{\mathrm{reg}}-\left\langle\partial_{\mu}\left(\sqrt{g} J^{\mu}\right)\right\rangle_{\mathrm{reg}} .
$$

The anomaly can be understood as a quantum violation of the expectation value of a classical identity even if it is not forced to be identically zero by a symmetry, as is case for the axial vector current with massive fermions.

In $D=4$, for example, the fields in the massless sector of each theory will exhibit an on-shell ${ }^{5}$ trace anomaly $[28,29]$

$$
\mathcal{A}^{\mathrm{W}}=A{\frac{1}{32 \pi^{2}}}^{*} R^{\mu \nu \rho \sigma *} R_{\mu \nu \rho \sigma}
$$

\footnotetext{
${ }^{5}$ That is to say ignoring the Ricci terms.
} 
so that in Euclidean signature

$$
\int_{X} d^{4} x \sqrt{g} \mathcal{A}^{\mathrm{W}}=A \chi(X)
$$

where $\chi(X)$ is the Euler characteristic of spacetime and $A$ is the anomaly coefficient, which for conformal field theories is related to the central charges $c$ and $a$ by $A=720(c-a)$. See table 1 for the anomaly coefficient contribution for each field.

In particular, $p$-form gauge fields $A_{p}$ in $D \neq 2 p+2$ provide nice examples of theories that are scale invariant but not conformal invariant. In $D=4 A_{p}$ and their duals $A_{(2-p)}$ yield $[4]$

$$
\int_{X} \mathcal{A}^{\mathrm{W}}\left(A_{2}\right)-\int_{X} \mathcal{A}^{\mathrm{W}}\left(A_{0}\right)=\chi(X)
$$

and

$$
\int_{X} \mathcal{A}^{\mathrm{W}}\left(A_{3}\right)=-2 \chi(X)
$$

These inequivalences were included in table 1. In arbitrary even dimensions

$$
\int_{X} \mathcal{A}^{\mathrm{W}}\left(A_{p}\right)-\int_{X} \mathcal{A}^{\mathrm{W}}\left(A_{\tilde{p}}\right)=(-1)^{p} \frac{1}{2}(p-\tilde{p}) \chi(X) .
$$

Such quantum inequivalence of $p$-forms and their duals has been called into question [33-35] on the grounds that their total stress tensors are the same and that the anomalous trace is unphysical. Nevertheless, the Euler characteristic factors they provide in the partition functions are important for the subjects of free energy [36] and entanglement anomalies [6]. This will the subject of section 5 .

\section{2 $D=10$ type IIA and $D=11$ M-theory: the roles of $\chi$ and $\rho$}

In this section, following [1-3], we focus on compactifications of M-theory and its low-energy limit $D=11$ supergravity on seven-manifolds $X^{7}$ with Betti numbers $b_{n}=b_{7-n}$. See table 1 . The compactifying manifolds we have in mind will be the product a Ricci flat manifold with special/exceptional holonomy and $b_{1}=0$, for example $G_{2}$-manifolds [19, 20,37], and an $n$-torus with $b_{1}=n$. Such compactifications preserve at least one Poincaré supersymmetry [17, 38, 39] as opposed to one AdS supersymmetry resulting from a squashed $S^{7}$ [40], for example, which has weak $G_{2}$ holonomy. The resulting field content is given in terms of the Betti numbers in table 1 in the case of $d=7$ dimensions. For example, the moduli space of torsion free $G_{2}$-structures is locally diffeomorphic to an open set of $H^{3}(X, \mathbb{R})$ and $b_{1}=0$, so we recover the familiar result for $G_{2}$ compactifications, with a single $D=4$ gravitino and $b_{3}$ geometric moduli (plus $b_{3}$ moduli arising from the M-theory 3 -form $A$ ). It holds similarly for $T^{7}, T^{3} \times K 3, \mathrm{CY} 3 \times S^{1}$, which covers all cases of concern.

Since

$$
\text { M on }\left(X^{6} \times S^{1}\right) \equiv \text { IIA on } X^{6}
$$

we can also read off the compactification of the Type IIA string and its low-energy limit $D=10$ IIA supergravity on six-manifolds $X^{6}$ with Betti numbers $c_{n}=c_{6-n}$, where

$$
\left(b_{0}, b_{1}, b_{2}, b_{3}\right)=\left(c_{0}, c_{0}+c_{1}, c_{1}+c_{2}, c_{2}+c_{3}\right)
$$


The number of degrees of freedom, $f$, and the anomaly coefficient $A$ for each massless supergravity field in $d=4[28,29]$ is given in table 1 . The total number of degrees of freedom is

$$
f=4\left(b_{0}+b_{1}+b_{2}+b_{3}\right)=4\left(2 c_{0}+2 c_{1}+2 c_{2}+c_{3}\right) .
$$

We have seen in the previous subsection that the spacetime Euler characteristic enters the anomaly calculation but more surprising perhaps is that the total coefficient $A$ can depend on the internal Euler characteristic:

$$
A=-\frac{1}{24} \chi\left(X^{6}\right)
$$

If we now combine 4.4 and 4.11 we are able to use the Künneth formula relating the Euler characteristics of even-dimensional compact manifolds

$$
\chi(X) \chi(Y)=\chi(X \times Y)
$$

to write $^{6}$

$$
\int_{X} \mathcal{A}^{\mathrm{W}}=-\frac{1}{24} \chi\left(X^{4}\right) \chi\left(X^{6}\right)=-\frac{1}{24} \chi\left(X^{4} \times X^{6}\right)
$$

Equally remarkable is that if we now repeat the calculation for $D=11$ supergravity on seven-manifolds $X^{7}$, the total $A$ coefficient depends on the internal $\rho\left(X^{7}\right)$ :

$$
A=-\frac{1}{24} \rho\left(X^{7}\right) \text {. }
$$

If we combine 4.4 and 1.9 and apply the odd dimensional analogue of the Künneth formula,

$$
\chi(X) \rho(Y)=\rho(X \times Y)
$$

we find ${ }^{7}$

$$
\int_{X} \mathcal{A}^{\mathrm{W}}=-\frac{1}{24} \chi\left(X^{4}\right) \rho\left(X^{7}\right)=-\frac{1}{24} \rho\left(X^{4} \times X^{7}\right)
$$

\subsection{Special case $X^{d}=X^{d-1} \times S^{1} ; \rho\left(X^{d}\right)=\chi\left(X^{d-1}\right)$}

The $d=4$ anomalies from compactification of Type IIA on $X^{6}$ and those from compactification of M-theory on $X^{7}$ must be compatible with the duality

$$
\text { M on }\left(X^{6} \times S^{1}\right) \equiv \text { IIA on } X^{6} \text {. }
$$

This is indeed the case as may be seen from table 1. The Betti numbers are related by 4.9 and hence

$$
\begin{aligned}
\rho\left(X^{7}\right) & =\chi\left(X^{6}\right), \\
\rho\left(X^{4} \times X^{7}\right) & =\chi\left(X^{4} \times X^{6}\right) .
\end{aligned}
$$

\footnotetext{
${ }^{6}$ We stress that this is not the $d=10$ anomaly but that of the massless fields after compactification to $d=4$.

${ }^{7}$ Once again we stress that this is not the $d=11$ anomaly but that of the massless fields after compactification to $d=4$. Indeed, the $d=11$ anomaly vanishes since $d$ is odd.
} 


\section{Partition functions}

\subsection{Topological quantum field theory}

The $\rho$-characteristic naturally appears in the log divergences of $p$-form partition functions $Z\left[A_{0}, A_{1}, \ldots\right]$. There is a long and rich history relating partition functions to topological invariants. See [41] for a review. Broadly speaking there are two classes of topological quantum field theories [42]: Schwarz-type and Witten-type. The Witten-type theories [43] have $Q$-exact actions, plus locally total derivative terms. Most directly relevant here, however, are the Schwarz-type topological quantum field theories [44, 45], which are defined to have topological classical actions that are not total derivatives, Chern-Simons theory being the best known example. Schwarz showed [44, 45] that a class of Schwarz-type $p$ form partition functions yield the Ray-Singer torsion, which, as we previously observed, is formally of the same structure as $\rho$. Schwarz's perspective was used in [46] to compute the Ray-Singer torsion of Lens spaces via Chern-Simons theory and generalised to $p$-form theories with non-trivial cohomology in [47], where the metric independence of the RaySinger torsion was re-derived using the path integral and BRST-framework.

Let us first review the Abelian Chern-Simons theory on a closed compact 3-manifold $X$,

$$
S_{\mathrm{CS}}[A]=\int_{M} A \wedge d A
$$

It is assumed, for now, that there are no zero-modes by considering $A$ valued in a flat $\pi_{1}(X)$-bundle $\mathcal{E}$ with trivial twisted Hodge-de Rham cohomology.

Applying Schwarz's geometric approach or the familiar BRST quantisation, see for example [47], the free energy is given by

$$
\begin{aligned}
F_{\mathrm{CS}}[A]:=\ln Z_{\mathrm{CS}}[A] & =-\frac{1}{4} \sum_{k=0}^{3}(-1)^{k} k \ln \operatorname{det}\left(\frac{\Delta_{k}}{\mu^{2}}\right) \\
& =-\frac{1}{4} \sum_{k=0}^{3}(-1)^{k} k \ln \operatorname{det}\left(\Delta_{k}\right)+\frac{1}{2} \ln \mu \sum_{k=0}^{3}(-1)^{k} k \operatorname{dim} \Delta_{k} .
\end{aligned}
$$

Here $\mu$ is a dimensionful parameter that enters through the measure, for example $D A=\prod_{n} \mu d a_{n}$, where $A=\sum a_{n} A^{(n)}$ for eigen-1-forms $A^{(n)}$ with eigenvalues $\lambda_{n}$ of the Laplacian $\Delta_{1}$.

As we have assumed there are no zero-modes, we can straightforwardly apply zetafunction regularisation of the partition function [48] with no further subtleties. As introduced by Ray and Singer [7] in the context of defining the Ray-Singer torsion, the zeta-regularised dimension and determinant of (twisted) Hodge-de Rahm Laplacians are defined by

$$
\left.\operatorname{dim} \Delta_{k}\right|_{\mathrm{reg}}:=\zeta_{k}(0),\left.\quad \ln \operatorname{det} \Delta_{k}\right|_{\mathrm{reg}}:=-\zeta_{k}^{\prime}(0),
$$

where

$$
\zeta_{k}^{\prime}(0)=\left.\frac{d}{d s} \zeta_{k}(s)\right|_{s=0}, \quad \zeta_{k}(s)=\sum_{n} \lambda_{n}^{-s}
$$


Since in odd dimensions $\zeta_{k}(0)=b_{k}$ we conclude

$$
F_{\mathrm{CS}}[A]=-\frac{1}{2}\left(\ln T_{\mathrm{RS}}(X, \mathcal{E})+\rho(M) \ln \mu\right),
$$

where $T_{\mathrm{RS}}(X, \mathcal{E})$ is the Ray-Singer torsion,

$$
T_{\mathrm{RS}}(X, \mathcal{E}):=\frac{1}{2} \sum_{k=0}^{d}(-1)^{k} k \zeta_{k}^{\prime}(0)
$$

Since the we assume a trivial twisted de Rham-Hodge cohomology $T_{\mathrm{RS}}(X, \mathcal{E})$ is metric independent [7].

Assuming the classically topological action implies metric independence, the logarithmic term, required on dimensional grounds, must be controlled by a topological invariant. This is familiar from the zeta regularisation of not necessarily topological field theories in even dimensions, where it is the Euler characteristic that appears, cf. [6]. Of course, the Euler characteristic is trivial in odd dimensions and we see that the $\rho$-characteristic takes its place.

We can generalise this observation to arbitrary dimensions via Abelian $B F$ theory

$$
S_{B F}[A, B]=\int_{M} B \wedge d A,
$$

where $B$ is a $p$-form and $A$ a $(d-p-1)$-form, possibly valued in a flat bundle $\mathcal{E}$. The action is invariant under the obvious local transformations,

$$
\delta B=d \Lambda_{p-1}, \quad \delta A=d \lambda_{d-p-2}
$$

with redundancies $\delta \Lambda_{p-1}=d \Lambda_{p-2}, \ldots$ and $\delta \lambda_{d-p-2}=d \lambda_{d-p-3}, \ldots, \delta \lambda_{0}=0$. The action is also invariant under the transformations

$$
\delta B=\Gamma_{p}, \quad \delta A=\gamma_{d-p-1}
$$

where $\Gamma, \gamma$ are harmonic forms, which can be used to trivialise the zero mode contributions to the partition function [47]. Note, one can alternatively keep the harmonics modes in the partition function explicitly [47].

The Schwarz resolvent methodology or BRST quantisation [47] yields the free energy,

$$
F_{B F}[A, B]=-\frac{1}{2} \sum_{k=0}^{d}(-1)^{k} k \operatorname{det} \frac{\Delta_{k}}{\mu^{2}}-\frac{1}{4} \sum_{k=0}^{d}(-1)^{k} \operatorname{det} \frac{\Delta_{k}}{\mu^{2}} .
$$

Applying zeta-regularisation we find,

$$
\begin{aligned}
F_{B F}[A, B]= & -T_{\mathrm{RS}}(M, \mathcal{E})+\ln \mu \sum_{k}^{d}(-1)^{k} k \zeta_{k}(0) \\
& -\frac{1}{4} \sum_{k=0}^{d}(-1)^{k} \zeta_{k}^{\prime}(0)+\frac{1}{2} \ln \mu \sum_{k=0}^{d}(-1)^{k} \zeta_{k}(0) .
\end{aligned}
$$


In odd dimensions, by Poincaré duality this reduces to,

$$
F_{B F}[A, B]=-T_{\mathrm{RS}}(M, \mathcal{E})-\rho(M) \ln \mu .
$$

In even dimensions $d=2 m$, we find

$$
F_{B F}[A, B]=0,
$$

where we have used $T_{\mathrm{RS}}(M, \mathcal{E})=0$, Poincaré duality and $\sum(-1)^{k} \zeta_{k}(0)=\sum\left(B_{d}^{(k)}-b_{k}\right)=0$, where $B_{i}^{(k)}$ are the integrated Seeley-DeWitt coefficients for $k$-forms (see section A for the $d=4$ case).

\subsection{Duality anomalies}

\subsubsection{Electromagnetic duality and the $\chi, \rho$ characteristics}

The $\rho$-characteristic also appears in the context of duality anomalies of not necessarily topological $p$-form field theories. Recall, although classically a free $p$-form is equivalent to its dual $\tilde{p}$-form, where $\tilde{p}=d-2-p$, this correspondence breaks down quantum mechanically [4]. In even dimensions there is a duality anomaly with consequences for physical properties, such as the entanglement entropy [6]. It is well-known that $\chi$, the Ray-Singer [7] and Reidmeister [49] torsions play important roles in the duality anomaly [6, 50]. Here we discuss the appearance of $\rho$ in this same context.

Let us consider the free energy of an Abelian $p$-form field $A$ and its dual $\tilde{A}$, with classical actions

$$
S_{p}=\frac{1}{2 e^{2}} \int_{X} F \wedge \star F, \quad \tilde{S}_{\tilde{p}}=\frac{1}{2 \tilde{e}^{2}} \int_{X} \tilde{F} \wedge \star \tilde{F},
$$

where $F=d A+\mathcal{F}, \tilde{F}=d \tilde{A}+\tilde{\mathcal{F}}$, where $\mathcal{F} \in 2 \pi H^{p+1}(X, \mathbb{Z})$. Here $X$ is a closed Riemannian manifold. The electric $e$ and magnetic $\tilde{e}$ charges satisfy $e \tilde{e}=2 \pi$ and have mass-dimensions

$$
[e]=p+1-d / 2=\frac{1}{2}(p-\tilde{p}), \quad[\tilde{e}]=-(p+1)+d / 2=\frac{1}{2}(\tilde{p}-p) .
$$

The electric potentials have mass-dimension $\left[A_{(p)}\right]=p$, so 1 -forms have the canonical geometric dimension, while the dual magnetic potentials have $\left[\tilde{A}_{(\tilde{p})}\right]=\tilde{p}=d-p-2$. This is consistent with dimensionless couplings for middle dimension field strengths.

The $p / \tilde{p}$ massless duality anomaly is defined by

$$
\mathcal{A}^{p, \tilde{p}}:=\ln \frac{Z_{p}}{Z_{\tilde{p}}}=F_{p}-F_{\tilde{p}},
$$

where $F_{p}=\ln Z_{p}$ is the free-energy.

We review here the derivation of the duality anomaly [6], which includes the non-zero mode contribution given in [50]. The partition function factors into three contributions,

$$
Z_{p}=Z_{p}^{\mathrm{osc}} Z_{p}^{\mathrm{zero}} Z_{p}^{\mathrm{inst}}
$$

corresponding to the oscillatory modes, $A \in \Omega_{\text {exact }}^{p}(X) \oplus \Omega_{\text {coexact }}^{p}(X)$, the zero modes, $\Delta A=0$, and the instantons, $\mathcal{F} \in 2 \pi H^{p+1}(X, \mathbb{Z})$. 
Oscillatory modes. Taking into account the ghost-for-ghosts [51], the partition function is formally given by

$$
Z_{p}^{\text {osc }}=\prod_{k=0}^{p}\left(\operatorname{det}^{\prime} \frac{\Delta_{p-k}}{\mu^{2}}\right)^{\frac{1}{2}(-1)^{k+1}(k+1)}=\prod_{k=0}^{p}\left(\operatorname{det}^{\prime} \frac{\Delta_{k}}{\mu^{2}}\right)^{\frac{1}{2}(-1)^{p+1-k}(p+1-k)},
$$

where $\Delta_{k}$ is the Hodge-de Rahm Laplacian, $\operatorname{det}^{\prime}$ denotes the restriction of the determinant to the subspace $\Omega_{\text {osc }}^{p}(X) \cong \Omega_{\text {exact }}^{p}(X) \oplus \Omega_{\text {coexact }}^{p}(X)$ of exact and coexact forms and $\mu$ is a mass-dimension-one constant required to make the path integral measure dimensionless.

The free energy has $\mu$-independent and $\ln \mu$-dependent terms,

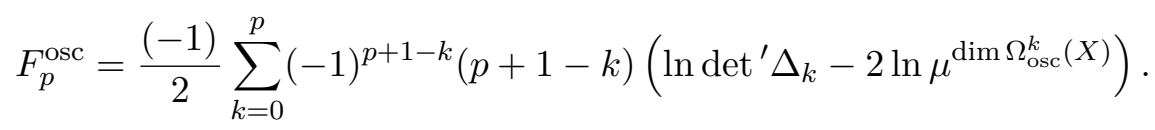

The $\operatorname{dim} \Omega_{\text {osc }}^{k}(X)$ and $\operatorname{det}^{\prime} \Delta_{k}$ appearing in (5.19) can be evaluated using zeta-regularisation,

$$
\left.\operatorname{dim} \Omega_{\mathrm{osc}}^{k}(X)\right|_{\mathrm{reg}}:=\zeta_{k}(0),\left.\quad \ln \operatorname{det}^{\prime} \Delta_{k}\right|_{\mathrm{reg}}:=\zeta_{k}^{\prime}(0),
$$

where

$$
\zeta_{k}^{\prime}(0)=\left.\frac{d}{d s} \zeta_{k}(s)\right|_{s=0}, \quad \zeta_{k}(s)=\sum_{\lambda>0} \lambda^{-s}
$$

and $\lambda$ are the non-zero eigenvalues of $\Delta_{k}$. Thus

$$
F_{p}^{\text {osc }}=-\frac{1}{2} \sum_{k=0}^{p}(-1)^{(p+1-k)}(p+1-k) \zeta_{k}^{\prime}(0)+\ln (\mu) \sum_{k=0}^{p}(-1)^{(p+1-k)}(p+1-k) \zeta_{k}(0) .
$$

The duality anomaly in the oscillatory mode sector is therefore given by $\mathcal{A}_{\text {osc }}^{p, \tilde{p}}=$ $\mathcal{A}_{\mathrm{osc}, \mathrm{const}}^{p, \tilde{p}}+\mathcal{A}_{\mathrm{osc}, \mu}^{p, \tilde{p}}$, where

$$
\begin{aligned}
\mathcal{A}_{\mathrm{osc}, \mathrm{const}}^{p, \tilde{p}} & =-\frac{1}{2} \sum_{k=0}^{d}(-1)^{(p+1-k)}(p+1-k) \zeta_{k}^{\prime}(0), \\
\mathcal{A}_{\mathrm{osc}, \mu}^{p, \tilde{p}} & =\ln (\mu) \sum_{k=0}^{d}(-1)^{(p+1-k)}(p+1-k) \zeta_{k}(0),
\end{aligned}
$$

and we have used Poincaré duality, $\zeta_{k}(s)=\zeta_{d-k}(s)$.

In even dimensions, $d=2 m$, it is straightforward to demonstrate $\mathcal{A}_{\text {osc }}^{p, \tilde{p}}=0$. From (5.23) we have

$$
\begin{aligned}
\mathcal{A}_{\mathrm{osc}, \mathrm{const}}^{p, \tilde{p}} & =-(-1)^{p+1}(p+1-m) \frac{1}{2} \sum_{k=0}^{d}(-1)^{k} \zeta_{k}^{\prime}(0), \\
\mathcal{A}_{\mathrm{osc}, \mu}^{p, \tilde{p}} & =\ln (\mu)(-1)^{p+1}(p+1-m) \sum_{k=0}^{d}(-1)^{k} \zeta_{k}(0) .
\end{aligned}
$$

Then the vanishing of $\mathcal{A}_{\mathrm{osc}}^{p, \tilde{p}}$ immediately follows from

$$
\sum_{k=0}^{d=2 m}(-1)^{k} k \zeta_{k}(s)=0
$$


which is essentially a consequence of $\Omega_{\text {osc }}^{p}(X) \cong \Omega_{\text {exact }}^{p}(X) \oplus \Omega_{\text {coexact }}^{p}(X)$ and implies the vanishing of the Ray-Singer torsion,

$$
\ln T_{\mathrm{RS}}(X)=\frac{1}{2} \sum_{k=0}^{d}(-1)^{k} k \zeta_{k}^{\prime}(0)
$$

in even dimensions [7]. Note, due to the non-trivial cohomology $T_{\mathrm{RS}}(X)$ is metric dependent. This can be rectified by defining $\hat{T}_{\mathrm{RS}}(X):=V_{H} \bullet(X) T_{\mathrm{RS}}(X)$, where $V_{H \bullet(X)}$ is an element of the cohomology determinant line, $\operatorname{det} H^{\bullet}(X):=\bigotimes_{k=0}^{d}\left(\operatorname{det} H^{k}(X)\right)^{(-1)^{k}}$, given by an arbitrary orthonormal basis in the cohomology with respect to the inner product induced by the representation of $H^{\bullet}(X)$ by harmonic forms and the Hodge $p$-form inner product on $\Omega^{\bullet}(X)$. For a nice introduction to these notions, see the proof of the CheegerMüller theorem [52, 53], using the Witten deformation of the Laplacian [54], given in [55].

From (5.23), for $d$ odd we have,

$$
\begin{aligned}
\mathcal{A}_{\mathrm{osc}, \mathrm{const}}^{p, \tilde{p}} & =(-1)^{p+1} \frac{1}{2} \sum_{k=0}^{d}(-1)^{k} k \zeta_{k}^{\prime}(0)=(-1)^{p+1} \ln T_{\mathrm{RS}}(X), \\
\mathcal{A}_{\mathrm{osc}, \mu}^{p, \tilde{p}} & =\ln (\mu)(-1)^{p} \sum_{k=0}^{d}(-1)^{k} k \zeta_{k}(0)=(-1)^{p+1} \ln (\mu) \rho(X) .
\end{aligned}
$$

We see that $\rho$ characterises the logarithmic contribution to the odd-dimensional oscillatory mode anomaly.

Zero modes. The action vanishes for zero-modes, so their contribution to the partition function is given solely by the volume of the space of flat connections modulo large gauge transformations, $A \sim A+2 \pi \theta$, where $\theta \in H^{p}(X, \mathbb{Z})$. The ghost $k<p$ forms contribute similarly. This gives

$$
Z_{p}^{\text {zero }}=\prod_{k=0}^{p} \operatorname{det}\left(\frac{2 \pi}{e^{2}} \mu^{2(k+1)} \Gamma_{p-k}\right)^{\frac{1}{2}(-1)^{k}}=\prod_{k=0}^{p} \operatorname{det}\left(\frac{2 \pi}{e^{2}} \mu^{2(p-k+1)} \Gamma_{k}\right)^{-\frac{1}{2}(-1)^{p+1-k}},
$$

where $\Gamma_{k}$ is the $b_{k}(X) \times b_{k}(X)$ Jacobian matrix corresponding to the change from the path integral measure basis to the topological basis of Free $H^{k}(X, \mathbb{Z})$.

Hence,

$$
F_{p}^{\text {zero }}=-\ln (\mu) \sum_{k=0}^{p}(-1)^{p+1-k}(p+1-k) b_{k}-\frac{1}{2} \sum_{k=0}^{p}(-1)^{p+1-k} \ln \operatorname{det}\left(\frac{2 \pi}{e^{2}} \Gamma_{k}\right)
$$

and

$$
\begin{aligned}
\mathcal{A}_{\mathrm{zero}, \text { const }}^{p, \tilde{p}} & =(-1)^{p} \frac{1}{2} \sum_{k=0}^{d}(-1)^{k} \ln \operatorname{det}\left(\frac{2 \pi}{e^{2}} \Gamma_{k}\right)+\frac{1}{2} \ln \operatorname{det}\left(\frac{2 \pi}{e^{2}} \Gamma_{p+1}\right) \\
& =(-1)^{p} \frac{1}{2} \ln \left(\frac{2 \pi}{e^{2}}\right) \chi(X)+(-1)^{p} \ln T_{\mathrm{R}}(X)+\frac{1}{2} \ln \operatorname{det}\left(\frac{2 \pi}{e^{2}} \Gamma_{p+1}\right), \\
\mathcal{A}_{\mathrm{zero}, \mu}^{p, \tilde{p}} & =\ln (\mu) \times\left\{\begin{array}{c}
\frac{(-1)^{p}}{2}(\tilde{p}-p) \chi(X) \text { even } \\
(-1)^{p} \rho(X) \quad \text { odd }
\end{array}\right.
\end{aligned}
$$




\begin{tabular}{|c|c|c|c|c|}
\hline Anomaly & $\begin{array}{l}\text { Massless } \\
\qquad d=2 n\end{array}$ & $\begin{array}{l}\text { Massless } \\
d=2 n+1\end{array}$ & $\begin{array}{l}\text { Massive } \\
\qquad d=2 n\end{array}$ & $\begin{array}{l}\text { Massive } \\
d=2 n+1\end{array}$ \\
\hline Oscillatory & 0 & $\begin{array}{l}(-1)^{p+1} \ln T_{\mathrm{RS}}(X) \\
+(-1)^{p+1} \rho(X) \ln \mu\end{array}$ & $\frac{(-1)^{p}}{2} \chi(M) \ln \left(\frac{\mu^{2}}{m^{2}}\right)$ & 0 \\
\hline Zero & $\begin{array}{c}(-1)^{p} \ln \left(\frac{e}{\tilde{e}}\right)^{\frac{\chi(X)}{2}}+\frac{1}{2} \operatorname{lndet}\left(\frac{2 \pi}{e^{2}} \Gamma_{p+1}\right) \\
+\frac{(-1)^{p}}{2}(\tilde{p}-p) \chi(X) \ln \mu\end{array}$ & $\begin{array}{c}(-1)^{p} \ln T_{\mathrm{R}}(X)+\frac{1}{2} \operatorname{lndet}\left(\frac{2 \pi}{e^{2}} \Gamma_{p+1}\right) \\
+(-1)^{p} \rho(X) \ln \mu\end{array}$ & 0 & 0 \\
\hline Instantons & $-\frac{1}{2} \ln \operatorname{det}\left(\frac{2 \pi}{e^{2}} \Gamma_{p+1}\right)$ & $-\frac{1}{2} \operatorname{lndet}\left(\frac{2 \pi}{e^{2}} \Gamma_{p+1}\right)$ & 0 & 0 \\
\hline Total & $\frac{(-1)^{p}}{2} \chi(X) \ln \frac{e \mu^{\tilde{p}}}{\tilde{\tilde{e}} \mu^{p}}$ & 0 & $\frac{(-1)^{p}}{2} \chi(M) \ln \left(\frac{\mu^{2}}{m^{2}}\right)$ & 0 \\
\hline
\end{tabular}

Table 7. The duality anomaly contributions for massless and massive $p$-forms.

where we have used $\left(\frac{2 \pi}{e^{2}} \Gamma_{k}\right)^{-1}=\frac{2 \pi}{\tilde{e}^{2}} S \Gamma_{d-k}$ for some unit determinant matrix $S$, and introduced the Reidemeister torsion,

$$
\ln T_{\mathrm{R}}(X)=\frac{1}{2} \sum_{k=0}^{d}(-1)^{k} \ln \operatorname{det}\left(\Gamma_{k}\right) .
$$

Instantons. The instanton contribution is given by the sum over field strengths belonging to $2 \pi H^{p+1}(m, \mathbb{Z})$, which we assume to be torsion free $H^{k}(m, \mathbb{Z}) \cong \mathbb{Z}^{b_{k}}$ so that

$$
Z_{p}^{\text {inst }}=\sum_{F \in \mathbb{Z}^{b} p+1} e^{-S[F]}
$$

In appendix A of [6] it is shown that

$$
Z_{\tilde{p}}^{\text {inst }}=\operatorname{det}\left(\frac{2 \pi}{e^{2}} \Gamma_{p+1}\right)^{1 / 2} Z_{p}^{\text {inst }}
$$

so

$$
\mathcal{A}_{\text {inst }}^{p, \tilde{p}}=-\frac{1}{2} \ln \operatorname{det}\left(\frac{2 \pi}{e^{2}} \Gamma_{p+1}\right) .
$$

Total. To summarise, the various contributions to the duality anomaly are given in table 7 . 
Note, the cancellation of the anomaly in odd dimensions makes use of the CheegerMüller theorem, which for torsion free cohomology reads

$$
\ln T_{\mathrm{RS}}(X, \mathcal{E})=\ln T_{\mathrm{R}}(X, \mathcal{E})
$$

In the case that there are no zero modes, the $\rho$-characteristic determines the $\ln \mu$ contribution to the anomaly.

Observe that the zero-mode contribution $\frac{(-1)^{p}}{2}(\tilde{p}-p) \chi(X) \ln \mu$ corresponds to the difference in the trace anomalies for $p$ - and $\tilde{p}$-forms [4], cf. (4.7). For even spheres, this agrees with the results of [36].

Torsion groups. Throughout we assumed the cohomology was torsion free. Relaxing this condition, $H^{k}(m, \mathbb{Z}) \cong \mathbb{Z}^{b_{k}} \oplus T^{k}$, the instanton partition function picks up a factor of $\left|T^{p+1}\right|$, while the zero-modes contribute $\prod_{k=0}^{p}\left|T^{k}\right|^{(-1)^{p+1-k}}[6]$. Hence, the anomaly due to the torsion groups is given by

$$
\begin{aligned}
A_{\mathrm{tor}}^{p, \tilde{p}} & =(-1)^{p+1} \sum_{k=0}^{p+1}(-1)^{k} \ln \left|T^{k}\right|-(-1)^{\tilde{p}+1} \sum_{k=0}^{\tilde{p}}(-1)^{k} \ln \left|T^{k}\right| \\
& =(-1)^{p+1} \sum_{k=0}^{d}(-1)^{k} \ln \left|T^{k}\right| .
\end{aligned}
$$

In even dimensions this is identically zero. In odd dimensions, it cancels against the difference between the Ray-Singer and Reidemeister torsions in the presence of torsion in the cohomology [53]

$$
\ln T_{\mathrm{RS}}(X)-\ln T_{\mathrm{R}}(X)=\sum_{k=0}^{d}(-1)^{k} \ln \left|T^{k}\right| .
$$

\subsubsection{Massive duality anomalies and Kaluza-Klein compactification}

Here we consider the vanishing massless $p=0, \tilde{p}=\hat{d}-2$ duality anomaly in $\operatorname{dim} \hat{X}=\hat{d}=$ $d+1=2 n+1$ dimensions and its Kaluza-Klein reduction on $\hat{X}=X \times S^{1}, \operatorname{dim} X=2 n$. In $\hat{d}$ dimensions we have,

$$
S_{0}=\frac{1}{2 \hat{e}^{2}} \int_{\hat{X}} \hat{F}_{(1)} \wedge \star \hat{F}_{(1)}, \quad \tilde{S}_{d-1}=\frac{1}{2 \hat{\tilde{e}}^{2}} \int_{\hat{X}} \hat{\tilde{F}}_{(d)} \wedge \star \hat{\tilde{F}}_{(d)}
$$

where the electric/magnetic charges satisfy $\hat{e} \hat{\tilde{e}}=2 \pi$. Compactifying on $\hat{X}=X \times S^{1}$, where $S^{1}$ has radius $R$, we have

$$
S_{0}=\frac{1}{2 e^{2}} \int_{X} F_{(1)} \wedge \star F_{(1)}+\frac{1}{2 e^{\prime 2}} \int_{X} F_{(0)} \wedge \star F_{(0)}+\sum_{n=-\infty}^{\infty} S_{0}^{(n)}
$$

where $S_{0}^{(n)} \sim \frac{1}{2 e^{2}} \int\left(F_{(1)}^{(n)} \wedge \star F_{(1)}^{(n)}+\frac{n^{2}}{2 R^{2}}\left(A_{(0)}^{(n)}\right)^{2}\right)$ correspond to the massive Kaluza-Klein scalars and

$$
e=\frac{\hat{e}}{\sqrt{2 \pi R}}, \quad e^{\prime}=\hat{e} \sqrt{2 \pi R}
$$


Similarly,

$$
\tilde{S}_{(d-1)}=\frac{1}{2 \tilde{e}^{\prime 2}} \int_{X} \tilde{F}_{(d)} \wedge \star \tilde{F}_{(d)}+\frac{1}{2 \tilde{e}^{2}} \int_{X} \tilde{F}_{(d-1)} \wedge \star \tilde{F}_{(d-1)}+\sum_{n=-\infty}^{\infty} S_{(d-1)}^{(n)}
$$

where

$$
\tilde{e}^{\prime}=\frac{\hat{\tilde{e}}}{\sqrt{2 \pi R}}, \quad \tilde{e}=\hat{\tilde{e}} \sqrt{2 \pi R}
$$

so that $e \tilde{e}=2 \pi$ and $e^{\prime} \tilde{e}^{\prime}=2 \pi$.

From the $d$-dimensional perspective the massless contribution to the duality anomaly is

$$
\frac{\chi(M)}{2}\left[\ln \left(\frac{e}{\tilde{e}}\right)-\ln \left(\frac{e^{\prime}}{\tilde{e}^{\prime}}\right)\right]+\chi(M) \ln (\mu)-2 \chi(M) \ln (\mu)=-\chi(M) \ln (2 \pi R \mu) .
$$

Since the $\hat{d}$-dimensional anomaly vanishes this must be cancelled by the duality anomaly of the infinite tower of massive Kaluza-Klein modes. The duality anomaly of massive $p$-forms was treated in [56]. In this case a $p$-form is classically dual to a $(d-p-1)$-form of the same mass, so that the difference for each $n$ of the massive 0 -forms and $(d-1)$-forms contributions in (5.39) and (5.41) to the free energy is the correct duality anomaly. In this case there are only the non-zero modes to contribute. In $d$-dimensions the $p, \tilde{p}=d-p-1$ duality anomaly with mass $m$ is given by,

$$
\mathcal{A}_{m}^{p, \tilde{p}}=(-1)^{p} \frac{1}{2} \sum_{k=0}^{d}(-1)^{k} \ln \operatorname{det}\left(\frac{\Delta_{k}+m^{2}}{\mu^{2}}\right) .
$$

Note, unlike the massless case there is no factor of $k$ in the sum - it is of 'Euler form' as opposed to 'Ray-Singer form' (of course, this distinction is only relevant in odd dimensions). Consequently, the anomaly vanishes in odd dimensions, due to Poincaré duality, but is nonzero in even dimensions, just as in the massless case. In the massless case the non-zero mode contribution to the anomaly in even dimensions vanished, whereas in the massive case it is non-vanishing and is the only contribution - there is nothing else for it to cancel against.

The determinant in (5.44) can be regulated using the spectral Hurwitz function. For a Laplace-type operator $A$ with non-negative spectrum $\{\lambda\}$ the spectral Hurwitz function is defined by the meromorphic continuation of

$$
\zeta_{A}(s, a):=\sum_{\lambda>0}(\lambda+a)^{-s}, \quad a>0 .
$$

Note, since the spectrum of $\Delta_{k}$ is non-negative we must include its zero-modes in the extended spectral Hurwitz function,

$$
Z_{k}(s, a):=\sum_{\lambda \in \operatorname{spec}\left(\Delta_{k}\right)}(\lambda+a)^{-s}=b_{k} a^{-s}+\zeta_{k}(s, a) .
$$

Then

$$
Z_{k}(0, a)=b_{k}+\zeta_{k}(0, a)
$$

and

$$
Z_{k}^{\prime}(s, a)=-b_{k} a^{-s} \ln a+\zeta_{k}^{\prime}(s, a)
$$


so that

$$
Z_{k}^{\prime}(0, a)=-b_{k} \ln a+\zeta_{k}^{\prime}(0, a) .
$$

We shall use $Z_{k}(s, a)$ to regulate (letting $a=m^{2}$ ) the determinant and dimension of $\Delta_{k}+a$ :

$$
Z_{k}(0, a)=:\left.\operatorname{dim}\left(\Delta_{k}+a\right)\right|_{\mathrm{reg}}, \quad Z_{k}^{\prime}(0, a)=:-\left.\ln \operatorname{det}\left(\Delta_{k}+a\right)\right|_{\mathrm{reg}}
$$

so that

$$
\begin{aligned}
\left.2(-1)^{p} \mathcal{A}_{m}^{p, \tilde{p}}\right|_{\text {reg }} & =\left.\sum_{k=0}^{d}(-1)^{k} \ln \operatorname{det}\left(\Delta_{k}+a\right)\right|_{\text {reg }}-\left.\ln \left(\mu^{2}\right) \sum_{k=0}^{d}(-1)^{k} \operatorname{dim}\left(\Delta_{k}+a\right)\right|_{\text {reg }} \\
& =-\sum_{k=0}^{d}(-1)^{k} Z_{k}^{\prime}(0, a)-\ln \mu^{2} \sum_{k=0}^{d}(-1)^{k} Z_{k}(0, a) \\
& =\chi(M) \ln a-\sum_{k=0}^{d}(-1)^{k} \zeta_{k}^{\prime}(0, a)-\chi(M) \ln \mu^{2}-\ln \mu^{2} \sum_{k=0}^{d}(-1)^{k} \zeta_{k}(0, a) \\
& =\chi(M) \ln \left(\frac{a}{\mu^{2}}\right)-\sum_{k=0}^{d}(-1)^{k} \zeta_{k}^{\prime}(0, a)-\ln \mu^{2} \sum_{k=0}^{d}(-1)^{k} \zeta_{k}(0, a)
\end{aligned}
$$

Noting that $\sum_{k=0}^{d}(-1)^{k} \zeta_{k}(s, a)=0$, cf. [57], we conclude

$$
\left.2(-1)^{p} \mathcal{A}_{m}^{p, \tilde{p}}\right|_{\text {reg }}=-\chi(M) \ln \left(\frac{\mu^{2}}{m^{2}}\right) .
$$

Hence, we obtain a non-zero massive $p$-form duality anomaly in even dimensions consistent with [56], but in contrast to [57]. The difference is due to our use of (5.46), as opposed to (5.45) applied in [57], for the regularisation. Its non-vanishing is required on physical grounds to cancel the massless anomaly in the Kaluza-Klein reduction, as we illustrate below.

The total anomaly for the $p=0, \tilde{p}=d-1$ Kaluza-Klein tower with masses $m_{n}=|n| / R$ is then

$$
\mathcal{A}_{\text {Kaluza-Klein }}^{p, \tilde{p}}=-\chi(M) \sum_{n=1}^{\infty} \ln \left(\frac{\mu R}{n}\right)=-\chi(M) \ln (\mu R) \sum_{n \in \mathbb{Z}_{\neq 0}} \frac{1}{|n|^{0}}+\chi(M) \sum_{n \in \mathbb{Z}_{\neq 0}} \ln |n| .
$$

Let us zeta-regulate the infinite sums,

$$
\left.\sum_{n \in \mathbb{Z}_{\neq 0}} \frac{1}{|n|^{0}}\right|_{\text {reg }}=2 \zeta(0)=-\left.1 \quad \sum_{n \in \mathbb{Z}_{\neq 0}} \ln |n|\right|_{\text {reg }}=-2 \zeta^{\prime}(0)=2 \ln \sqrt{2 \pi} .
$$

Hence,

$$
\mathcal{A}_{\text {Kaluza-Klein }}^{p, \tilde{p}}=\chi(M) \ln (\mu R)+2 \chi(M) \ln \sqrt{2 \pi}=\chi(M) \ln (2 \pi \mu R)
$$

and the massless $d$-dimensional duality anomaly is cancelled precisely by the duality anomalies of the massive Kaluza-Klein tower.

Of course, the cancellation between the massless and massive duality anomalies witnessed above had to be the case; the $\hat{d}$-dimensional anomaly is always vanishing. 
If one insists that type IIA supergravity is duality anomaly free, then the accompanying massive tower of states is essential. This provides yet another rational for the inclusion of D-branes. Equivalently, it provides yet another rational for M-theory. The $D=11$ theory is duality anomaly free and so its compactification to type IIA must be duality anomaly free. Duality anomaly freedom is ensured by M-theory. The type IIA R-R 1form potential derives from the metric, so really this argument assumes duality anomaly freedom for gravity. Since gravity is non-Abelian, unlike the R-R sector, it is not clear that gravitational duality can be implemented. At least in the free case, however, classically dual graviton theories are well-known, cf. for example [58-60], and one can at least test the duality anomaly freedom for the free theory. Note, from the point of view of $E_{11}$ [61] there should be, and there is [62], an interacting dual graviton theory and consistency of M-theory would imply duality anomaly freedom.

\section{Conclusions}

In this paper the topological invariant

$$
\rho(X)=7 b_{0}-5 b_{1}+3 b_{2}-b_{3}
$$

introduced in [1-3] to describe the compactification of M-theory from $D=11$ to $D=4$, is generalized to arbitrary odd dimensions $\operatorname{dim} X=2 m+1$,

$$
\rho(X)=\sum_{p=0}^{m}(-1)^{p}(\operatorname{dim} X-2 p) b_{p}(X),
$$

and shown in several respects to behave as an odd-dimensional analogue of the Euler characteristic in even dimensions. For example it obeys the Künneth-type formula,

$$
\rho(X \times Y)=\rho(X) \chi(Y),
$$

where $X$ and $Y$ are even and odd dimensional, respectively, and is the unique linear combination of betti numbers to do so.

Whereas $\chi$ is related to the Poincare polynomial $P(z)$ by $P(-1), \rho$ is given by $P^{\prime}(-1)$. Both vanish for group manifolds, except for $\rho(\mathrm{U}(1))=1$ and $\rho(\mathrm{SU}(2))=3$. For cosets $G / H$ on the other hand

$$
\chi(G / H)=-\prod_{i=1}^{r} \frac{g_{i}+1}{h_{i}+1},
$$

when $r:=\operatorname{rank} G=s:=\operatorname{rank} H$, and

$$
\rho(G / H)=g_{s+1} \prod_{i=1}^{s} \frac{g_{i}+1}{h_{i}+1} .
$$

when $r=s+1$, where $g_{i}=2 c(G)_{i}-1, h_{i}=2 c(H)_{i}-1$, with $c(G)_{i}$ and $c(H)_{i}$ the degree of the $i^{\text {th }}$ Casimir invariant of $G$ and $H$, respectively. For example $\chi\left(S^{2 n}\right)=2, \rho\left(S^{2 n}\right)=$ $2 n ; \chi\left(S^{2 n+1}\right)=0, \rho\left(S^{2 n+1}\right)=2 n+1$. 
The $\rho$-characteristic also makes an appearance in the $d=4$ Weyl anomaly of the massless sector of M-theory compactifications. For $d=11$ M-theory on $X^{4}$ (spacetime) $\times$ $Y^{7}$ (internal) the $d=4$ on-shell Weyl anomaly $\mathcal{A}^{\mathrm{W}}$ is given by

$$
\int \mathcal{A}^{\mathrm{W}}=-\frac{1}{24} \chi\left(X^{4}\right) \rho\left(Y^{7}\right)=-\frac{1}{24} \rho\left(X^{4} \times Y^{7}\right)
$$

on using the Künneth rule (1.4) and hence vanishes when $Y^{7}$ (and therefore $X^{11}$ ) is selfmirror. By virtue of the special case (1.5), this is entirely consistent with the equivalence

$$
\text { M-theory on } X^{10} \times S^{1} \equiv \text { IIA on } X^{10} \text {. }
$$

Odd dimensional generalized mirror maps are characterised by $\rho$, where $\rho \mapsto(-)^{m} \rho$ for $d=2 m+1$, in analogy to $\chi$ for even dimensions. If $\rho=0$ the manifold is 'self-mirror' and for the case of $G_{2}$ manifolds this defines an axis of symmetry [1, 19, 20]. Conventional mirror symmetry relates theories with Ricci-flat manifolds of special holonomy $X$, for which $b_{1}=0$, for example Calabi-Yau, $G_{2}$, Spin(7). Generalized mirror symmetry permits nonvanishing $b_{1}$, for example $\left(X \times T^{k}\right) / \Gamma$ with $b_{1}=k$, which in fact exhaust all closed Ricci-flat manifolds preserving some supersymmetry.

The $\rho$-characteristic naturally appears in the log divergences of $p$-form partition functions $Z\left[A_{0}, A_{1}, \ldots\right]$ in odd dimensions. We find

$$
F_{\mathrm{CS}}[A]=-\frac{1}{2}\left(\ln T_{\mathrm{RS}}(M, \mathcal{E})+\rho(M) \ln \mu\right),
$$

where $T_{\mathrm{RS}}(M, \mathcal{E})$ is the Ray-Singer torsion, in the case of Chern-Simons and

$$
F_{B F}[A, B]=-T_{\mathrm{RS}}(M, \mathcal{E})-\rho(M) \ln \mu .
$$

in the case of $B F$.

Since the Weyl anomaly arises because the operations of regularizing and taking the trace do not commute, the anomaly $\mathcal{A}^{\mathrm{W}}$ in a theory which is not classically Weyl invariant may be defined as in (4.1). Recall, although classically a free $p$-form is equivalent to its dual $\tilde{p}$-form, where $\tilde{p}=d-2-p$, this correspondence breaks down quantum mechanically [4]. In the case of p-forms, there is a difference between the partition function of a $p$-form and its dual and hence between $\mathcal{A}^{W}$ and its dual [4]: in arbitrary even dimensions

$$
\int_{X} \mathcal{A}^{\mathrm{W}}\left(A_{p}\right)-\int_{X} \mathcal{A}^{\mathrm{W}}\left(A_{\tilde{p}}\right)=(-1)^{p} \frac{1}{2}(p-\tilde{p}) \chi(X) .
$$

Such quantum inequivalence of $p$-forms and their duals also appears in the context of duality anomalies shown in table 7 . The $\rho$-characteristic also appears in the oscillitary mode sum but is exactly cancelled by the zero modes. There is no duality anomaly in odd dimensions but we noted

$$
\mathcal{A}_{\text {zero }, \mu}^{p, \tilde{p}}=\ln (\mu) \times\left\{\begin{array}{cc}
\frac{(-1)^{p}}{2}(\tilde{p}-p) \chi(X) & \text { even } \\
(-1)^{p} \rho(X) & \text { odd }
\end{array}\right.
$$

so, in this sense, $(-1)^{p} \rho(X)$ play the role in odd dimensions that Weyl anomaly does in even. For conformal field theories in even dimensions the $a$-theorem [63-65] is governed 
by the coefficient of $\chi$. So one might be tempted to think that in odd dimensions the $F$-theorem [66-69] would be governed by the coefficent of $\rho$. This is not apparent in the $F$-theorem literature, which is, however, confined to spheres. Perhaps the free energy of non-spherical manifolds will throw more light on this.

In the case of Kaluza-Klein, the vanishing of the duality anomaly in odd dimensions means that, when compactified to an even dimension, the contribution of the massless modes must be exactly cancelled by the infinite tower of massive KK modes, as is the case for the axial anomaly $[25,26]$. This has interesting implications for string/M theory: assuming that by virtue of its odd dimensionality M-theory has no anomaly, ${ }^{8}$ the $D=10$ Type IIA string requires the infinite tower of massive D0-branes, in other words, the eleventh dimension of M-theory!

\section{Acknowledgments}

We are grateful to Philip Candelas and Xenia del la Ossa for helpful discussions and comments on the manuscript. LB is supported by the Leverhulme Research Project Grant RPG-2018-329. MJD is grateful to Marlan Scully for his hospitality in the Institute for Quantum Science and Engineering, Texas A\&M University, and to the Hagler Institute for Advanced Study at Texas A\&M for a Faculty Fellowship. The work of MJD was supported in part by the STFC under rolling grant ST/P000762/1. SN is supported by STFC grant $\mathrm{ST} / \mathrm{T} 000686 / 1$.

\section{A $\quad B_{4}$ coefficients for $p$-forms}

Here we summarise some of the Weyl anomaly coefficient data for $p$-forms, see for example [70] for more details. The Weyl anomaly is given by

$$
(4 \pi)^{2} \mathcal{A}^{\mathrm{W}}=c F-a G+e R^{2}+f \nabla^{2} R+g R^{*} R
$$

where

$$
\begin{aligned}
& G=R_{\mu \nu \rho \sigma} R^{\mu \nu \rho \sigma}-4 R_{\mu \nu} R^{\mu \nu}+R^{2} \\
& F=C^{\mu \nu \rho \sigma} C_{\mu \nu \rho \sigma}
\end{aligned}
$$

and where $C_{\mu \nu \rho \sigma}$ is the Weyl tensor.

Note massless representations on $Y=X \times S^{1}$ with Betti numbers $b_{k}$ correspond to massive representations on $X$ with Betti numbers $c_{k}$

$$
\left(b_{0}, b_{1}, b_{2}, b_{3}, b_{4}, b_{5}\right)=\left(c_{0}, c_{1}+c_{0}, c_{2}+c_{1}, c_{3}+c_{2}, c_{4}+c_{3}, c_{4}\right)
$$

\footnotetext{
${ }^{8}$ The R-R 1-form potential derives from the metric in $D=11$, so really this argument assumes duality anomaly freedom for gravity.
} 


$\begin{array}{lrrrrrr}A_{p} & r e p & \bar{a} & \bar{c} & \bar{e} & \bar{f} & \bar{g} \\ & & & & & & \\ A_{0} & (0,0) & 2 & 6 & 10 & 24 & 0 \\ A_{1} & (1 / 2,1 / 2) & 128 & 84 & 20 & -24 & 0 \\ A_{2}{ }^{+} & (1,0) & -54 & 78 & 10 & -48 & 120 \\ A_{2}{ }^{-} & (0,1) & -54 & 78 & 10 & -48 & -120 \\ A_{3} & (1 / 2,1 / 2) & 128 & 84 & 20 & -24 & 0 \\ A_{4} & (0,0) & 2 & 6 & 0 & 24 & 0 \\ & & & & & & \\ E=A_{2}-2 A_{1}+2 A_{0} & 360 & -360 & 0 & 0 & 0 & 0 \\ T=A_{2}{ }^{+}-A_{2}{ }^{-} & 0 & 0 & 0 & 0 & 0 & 240 \\ (E \pm T) / 2=A_{2}{ }^{+}-A_{1}+A_{0} & 180 & -180 & 0 & 0 & 0 & 0 \\ A_{0}-A_{4} & 0 & 0 & 0 & 0 & 0 & 0 \\ A_{1}-A_{3} & 0 & 0 & 0 & 0 & 0 & 0\end{array}$

Table 8. $p$-form $A_{p}$ anomaly $\mathrm{SO}(4)$ representations in $d=4$. Here $E$ denotes the Euler characteristic combination and $T$ the Hirzebruch signature combination. In the final two rows the naïvely dual potentials are compared to emphasise their equivalence. Note, $\bar{x}=x / 720$.

$$
\begin{array}{lrrrrr}
B_{p} & r e p & a & c & e & f \\
B_{0}=A_{0} & 1 & 2 & 6 & 10 & 24 \\
B_{1}=A_{1}-A_{0} & 3 & 126 & 78 & 10 & -48 \\
B_{2}=A_{2}-A_{1}+A_{0} & 3 & -234 & 78 & 10 & -48 \\
B_{3}=A_{3}-A_{2}+A_{1}-A_{0} & 1 & 362 & 6 & 10 & 24 \\
B_{4}=A_{4}-A_{3}+A_{2}-A_{1}+A_{0} & 0 & -360 & 0 & 0 & 0 \\
& & & & & \\
B_{3}-B_{0}=E & 0 & -360 & 0 & 0 & 0 \\
B_{2}-B_{1}=-E & 0 & -360 & 0 & 0 & 0 \\
B_{4}=-E & 0-360 & 0 & 0 & 0
\end{array}
$$

Table 9. $p$-form $A_{p}$ anomaly massive $\mathrm{SO}(3)$ representations $B_{p}$ in $d=4$. The final three rows display the $(p, \tilde{p})=(2,1),(p, \tilde{p})=(3,0)$ and $(p, \tilde{p})=(4,-1)$ massive Weyl anomaly inequivalences, respectively. (Here, $p<0$ represents a formal form with vanishing anomaly coefficients.) 


$$
\begin{array}{lrrrrrr}
C_{p} & r e p & a & c & e & f & g \\
C_{0}=A_{0} & 1 & 2 & 6 & 10 & 24 & 0 \\
C_{1}=A_{1}-2 A_{0} & 2 & 124 & 72 & 0 & -72 & 0 \\
C_{2}=A_{2}-2 A_{1}+3 A_{0} & 1 & -358 & 6 & 10 & 24 & 0 \\
C_{3}=A_{3}-2 A_{2}+3 A_{1}-4 A_{0} & 0 & 720 & 0 & 0 & 0 & 0 \\
C_{4}=A_{4}-2 A_{3}+3 A_{2}-4 A_{1}+5 A_{0} & 0 & -1080 & 0 & 0 & 0 & 0 \\
& & & & & & \\
C_{2}-C_{0}=E & 0 & -360 & 0 & 0 & 0 & 0 \\
C_{3}=-2 E & 0 & -720 & 0 & 0 & 0 & 0 \\
C_{4}=3 E & 0 & -1080 & 0 & 0 & 0 & 0 \\
\left(C_{2}-C_{0}\right)^{ \pm}=\frac{1}{2}(E \pm T) & 0 & -180 & 0 & 0 & 0 \pm 120
\end{array}
$$

Table 10. $p$-form $A_{p}$ anomaly massless $\mathrm{SO}(2)$ representations $C_{p}$ in $d=4$. The final four rows display the $(p, \tilde{p})=(2,0),(p, \tilde{p})=(3,-1),(p, \tilde{p})=(4,-2)$ massless Weyl anomaly inequivalences and the splitting into (anti)self-dual parts.

$$
\begin{array}{ll}
p & =c_{0} \\
0 b_{0} & =-c_{0}+c_{1} \\
1 b_{1}-2 b_{0} & =c_{0}-c_{1}+c_{2} \\
2 b_{2}-2 b_{1}+3 b_{0} & =-c_{0}+c_{1}-c_{2}+c_{3} \\
3 b_{3}-2 b_{2}+3 b_{1}-4 b_{0} & c_{0}-c_{1}+c_{2}-c_{3}+c_{4} \\
4 b_{4}-2 b_{3}+3 b_{2}-4 b_{1}+5 b_{0} & =
\end{array}
$$

where we see the 4-forms have $\rho(Y)$ and $\chi(X)$ degrees of freedom, respectively.

Since $\rho(Y)=\chi(X)$, the massive Weyl anomaly inequivalences in $d=4$

$$
\begin{aligned}
\int_{X} \mathcal{A}^{\mathrm{W}}\left(A_{2}\right)-\int_{X} \mathcal{A}^{\mathrm{W}}\left(A_{1}\right) & =\chi(X), \\
\int_{X} \mathcal{A}^{\mathrm{W}}\left(A_{3}\right)-\int_{X} \mathcal{A}^{\mathrm{W}}\left(A_{0}\right) & =-\chi(X), \\
\int_{X} \mathcal{A}^{\mathrm{W}}\left(A_{4}\right) & =\chi(X),
\end{aligned}
$$

where in general

$$
\int_{X} \mathcal{A}^{\mathrm{W}}\left(A_{p}\right)=\sum_{k=0}^{p}(-1)^{k} c_{p-k},
$$

follow from the zero-modes ${ }^{9}$ contributions to the vanishing massless inequivalences in $d=5$,

\footnotetext{
${ }^{9}$ Since the zero-mode and oscillatory contributions cancel in odd dimensions, one could equivalently use the oscillatory modes.
} 
when compactified on a circle

$$
\begin{aligned}
\left.\int_{Y} \mathcal{A}^{\mathrm{W}}\left(A_{2}\right)\right|_{\text {zero }}-\left.\int_{Y} \mathcal{A}^{\mathrm{W}}\left(A_{1}\right)\right|_{\text {zero }} & =\rho(Y), \\
\left.\int_{Y} \mathcal{A}^{\mathrm{W}}\left(A_{3}\right)\right|_{\text {zero }}-\left.\int_{Y} \mathcal{A}^{\mathrm{W}}\left(A_{0}\right)\right|_{\text {zero }} & =-\rho(Y), \\
\left.\int_{Y} \mathcal{A}^{\mathrm{W}}\left(A_{5}\right)\right|_{\text {zero }} & =\rho(Y),
\end{aligned}
$$

where in general

$$
\left.\int_{Y} \mathcal{A}^{\mathrm{W}}\left(A_{p}\right)\right|_{\text {zero }}=\sum_{k=0}^{p}(-1)^{k}(k+1) b_{p-k} .
$$

Open Access. This article is distributed under the terms of the Creative Commons Attribution License (CC-BY 4.0), which permits any use, distribution and reproduction in any medium, provided the original author(s) and source are credited.

\section{References}

[1] M.J. Duff and S. Ferrara, Generalized mirror symmetry and trace anomalies, Class. Quant. Grav. 28 (2011) 065005 [arXiv: 1009.4439] [INSPIRE].

[2] M.J. Duff and S. Ferrara, Four curious supergravities, Phys. Rev. D 83 (2011) 046007 [arXiv: 1010.3173] [INSPIRE].

[3] M.J. Duff, S. Ferrara and A. Marrani, D = 3 unification of curious supergravities, JHEP 01 (2017) 023 [arXiv: 1610.08800] [INSPIRE].

[4] M.J. Duff and P. van Nieuwenhuizen, Quantum inequivalence of different field representations, Phys. Lett. B 94 (1980) 179 [INSPIRE].

[5] E. Witten, On S duality in Abelian gauge theory, Selecta Math. 1 (1995) 383 [hep-th/9505186] [INSPIRE].

[6] W. Donnelly, B. Michel and A. Wall, Electromagnetic duality and entanglement anomalies, Phys. Rev. D 96 (2017) 045008 [arXiv:1611.05920] [INSPIRE].

[7] $R$ torsion and the Laplacian on Riemannian manifolds, Adv. Math. 7 (1971) 145 [InSPIRE].

[8] S. Cecotti, P. Fendley, K.A. Intriligator and C. Vafa, A new supersymmetric index, Nucl. Phys. B 386 (1992) 405 [hep-th/9204102] [INSPIRE].

[9] J. Roberts, Unusual formulae for the Euler characteristic, J. Knot Theor. Ramificat. 11 (2002) 793.

[10] L. Yu, A property that characterizes euler characteristic among invariants of combinatorial manifolds, Adv. Math. 225 (2010) 794.

[11] W. Greub, S. Halperin and R. Vanstone, Connections, curvature, and cohomology V3: cohomology of principal bundles amd homogeneous spaces, volume 47, part C, Academic press, (1976).

[12] P. Candelas, M. Lynker and R. Schimmrigk, Calabi-Yau manifolds in weighted $P_{4}$, Nucl. Phys. B 341 (1990) 383 [INSPIRE].

[13] B.R. Greene and M.R. Plesser, (2,2) and (2,0) superconformal orbifolds, HUTP-89-A-043, (1989). 
[14] B.R. Greene and M.R. Plesser, Duality in Calabi-Yau moduli space, Nucl. Phys. B 338 (1990) 15 [INSPIRE].

[15] P.S. Aspinwall, C.A. Lütken and G.G. Ross, Construction and couplings of mirror manifolds, Phys. Lett. B 241 (1990) 373 [inSPIRE].

[16] P. Candelas, X.C. De La Ossa, P.S. Green and L. Parkes, A pair of Calabi-Yau manifolds as an exactly soluble superconformal theory, Nucl. Phys. B 359 (1991) 21 [AMS/IP Stud. Adv. Math. 9 (1998) 31] [INSPIRE].

[17] S.L. Shatashvili and C. Vafa, Superstrings and manifold of exceptional holonomy, Selecta Math. 1 (1995) 347 [hep-th/9407025] [INSPIRE].

[18] B.S. Acharya, Dirichlet Joyce manifolds, discrete torsion and duality, Nucl. Phys. B 492 (1997) 591 [hep-th/9611036] [INSPIRE].

[19] D.D. Joyce, Compact Riemannian 7-manifolds with holonomy $G_{2}$. I, J. Diff. Geom. 43 (1996) 291.

[20] D.D. Joyce, Compact Riemannian 7-manifolds with holonomy $G_{2}$. II, J. Diff. Geom. 43 (1996) 329.

[21] A.P. Braun, S. Majumder and A. Otto, On mirror maps for manifolds of exceptional holonomy, JHEP 10 (2019) 204 [arXiv:1905.01474] [INSPIRE].

[22] A.E. Fischer and J.A. Wolf, The structure of compact Ricci-flat Riemannian manifolds, J. Diff. Geom. 10 (1975) 277.

[23] P.S. Aspinwall and D.R. Morrison, String theory on K3 surfaces, AMS/IP Stud. Adv. Math. 1 (1996) 703 [hep-th/9404151] [INSPIRE].

[24] E. Bergshoeff, R. Kallosh, T. Ortín, D. Roest and A. Van Proeyen, New formulations of $D=10$ supersymmetry and D8-O8 domain walls, Class. Quant. Grav. 18 (2001) 3359 [hep-th/0103233] [INSPIRE].

[25] M.J. Duff and D.J. Toms, Divergences and anomalies in Kaluza-Klein theories, in Quantum gravity, Springer, Boston, MA, U.S.A. (1984), pg. 431.

[26] M.J. Duff and D.J. Toms, Kaluza-Klein-kounterterms, in Unification of fundamental particle interactions II, Springer, Boston, MA, U.S.A. (1983), pg. 29.

[27] S. Deser, M.J. Duff and C.J. Isham, Nonlocal conformal anomalies, Nucl. Phys. B 111 (1976) 45 [INSPIRE].

[28] M.J. Duff, Observations on conformal anomalies, Nucl. Phys. B 125 (1977) 334 [InSPIRE].

[29] M.J. Duff, Twenty years of the Weyl anomaly, Class. Quant. Grav. 11 (1994) 1387 [hep-th/9308075] [INSPIRE].

[30] L. Casarin, H. Godazgar and H. Nicolai, Conformal anomaly for non-conformal scalar fields, Phys. Lett. B $\mathbf{7 8 7}$ (2018) 94 [arXiv:1809.06681] [INSPIRE].

[31] T. Kimura, Divergence of axial-vector current in the gravitational field, Prog. Theor. Phys. 42 (1969) 1191 [INSPIRE].

[32] R. Delbourgo and A. Salam, The gravitational correction to PCAC, Phys. Lett. B 40 (1972) 381 [INSPIRE].

[33] W. Siegel, Quantum equivalence of different field representations, Phys. Lett. B 103 (1981) 107 [INSPIRE]. 
[34] M.T. Grisaru, N.K. Nielsen, W. Siegel and D. Zanon, Energy momentum tensors, supercurrents, (super)traces and quantum equivalence, Nucl. Phys. B 247 (1984) 157 [INSPIRE].

[35] Z. Bern, C. Cheung, H.-H. Chi, S. Davies, L. Dixon and J. Nohle, Evanescent effects can alter ultraviolet divergences in quantum gravity without physical consequences, Phys. Rev. Lett. 115 (2015) 211301 [arXiv: 1507.06118] [INSPIRE].

[36] H. Raj, A note on the sphere free energy of p-form gauge theory and Hodge duality, Class. Quant. Grav. 34 (2017) 247001 [arXiv: 1611.02507] [INSPIRE].

[37] G. Papadopoulos and P.K. Townsend, Compactification of D $=11$ supergravity on spaces of exceptional holonomy, Phys. Lett. B 357 (1995) 300 [hep-th/9506150] [InSPIRE].

[38] M.J. Duff, B.E.W. Nilsson and C.N. Pope, Compactification of $d=11$ supergravity on $K 3 \times T^{3}$, Phys. Lett. B 129 (1983) 39 [inSPIRE].

[39] M.J. Duff, Kaluza-Klein theories and superstrings, in Les Houches school of theoretical physics: architecture of fundamental interactions at short distances, (1985).

[40] M.A. Awada, M.J. Duff and C.N. Pope, $N=8$ supergravity breaks down to $N=1$, Phys. Rev. Lett. 50 (1983) 294 [INSPIRE].

[41] D. Birmingham, M. Blau, M. Rakowski and G. Thompson, Topological field theory, Phys. Rept. 209 (1991) 129 [INSPIRE].

[42] D. Birmingham, M. Rakowski and G. Thompson, Renormalization of topological field theory, Nucl. Phys. B 329 (1990) 83 [inSPIRE].

[43] E. Witten, Topological quantum field theory, Commun. Math. Phys. 117 (1988) 353 [INSPIRE].

[44] A.S. Schwarz, The partition function of degenerate quadratic functional and Ray-Singer invariants, Lett. Math. Phys. 2 (1978) 247 [InSPIRE].

[45] A.S. Schwarz, The partition function of a degenerate functional, Commun. Math. Phys. 67 (1979) 1 [INSPIRE].

[46] C. Nash and D.J. O'Connor, Determinants of Laplacians, the Ray-Singer torsion on lens spaces and the Riemann zeta function, J. Math. Phys. 36 (1995) 1462 [Erratum ibid. 36 (1995) 4549] [hep-th/9212022] [INSPIRE].

[47] M. Blau and G. Thompson, Topological gauge theories of antisymmetric tensor fields, Annals Phys. 205 (1991) 130 [INSPIRE].

[48] S.W. Hawking, Zeta function regularization of path integrals in curved space-time, Commun. Math. Phys. 55 (1977) 133 [INSPIRE].

[49] K. Reidemeister, Homotopieringe und Linsenräume (in German), Abh. Math. Semin. Univ. Hambg. 11 (1935) 102.

[50] A.S. Schwarz and Y.S. Tyupkin, Quantization of antisymmetric tensors and Ray-Singer torsion, Nucl. Phys. B 242 (1984) 436 [inSPIRE].

[51] W. Siegel, Hidden ghosts, Phys. Lett. B 93 (1980) 170 [InSPIRE].

[52] W. Müller, Analytic torsion and r-torsion of Riemannian manifolds, Adv. Math. 28 (1978) 233.

[53] J. Cheeger, Analytic torsion and the heat equation, Ann. Math. 109 (1979) 259. 
[54] E. Witten, Supersymmetry and Morse theory, J. Diff. Geom. 17 (1982) 661.

[55] M. Braverman, New proof of the Cheeger-Müller theorem, Ann. Global Anal. Geom. 23 (2003) 77.

[56] S.M. Kuzenko and K. Turner, Effective actions for dual massive (super) p-forms, JHEP 01 (2021) 040 [arXiv: 2009. 08263] [INSPIRE].

[57] I.L. Buchbinder, E.N. Kirillova and N.G. Pletnev, Quantum equivalence of massive antisymmetric tensor field models in curved space, Phys. Rev. D 78 (2008) 084024 [arXiv: 0806.3505] [INSPIRE].

[58] C.M. Hull, Duality in gravity and higher spin gauge fields, JHEP 09 (2001) 027 [hep-th/0107149] [INSPIRE].

[59] P. de Medeiros and C. Hull, Exotic tensor gauge theory and duality, Commun. Math. Phys. 235 (2003) 255 [hep-th/0208155] [INSPIRE].

[60] P. de Medeiros and C. Hull, Geometric second order field equations for general tensor gauge fields, JHEP 05 (2003) 019 [hep-th/0303036] [INSPIRE].

[61] P.C. West, $E_{11}$ and M-theory, Class. Quant. Grav. 18 (2001) 4443 [hep-th/0104081] [INSPIRE].

[62] K. Glennon and P. West, The non-linear dual gravity equation of motion in eleven dimensions, Phys. Lett. B 809 (2020) 135714 [arXiv:2006. 02383] [InSPIRE].

[63] J.L. Cardy, Is there a $c$ theorem in four-dimensions?, Phys. Lett. B 215 (1988) 749 [InSPIRE].

[64] H. Osborn, Derivation of a four-dimensional c theorem, Phys. Lett. B 222 (1989) 97 [INSPIRE].

[65] Z. Komargodski and A. Schwimmer, On renormalization group flows in four dimensions, JHEP 12 (2011) 099 [arXiv:1107.3987] [INSPIRE].

[66] R.C. Myers and A. Sinha, Seeing a c-theorem with holography, Phys. Rev. D 82 (2010) 046006 [arXiv: 1006.1263] [INSPIRE].

[67] H. Casini, M. Huerta and R.C. Myers, Towards a derivation of holographic entanglement entropy, JHEP 05 (2011) 036 [arXiv:1102.0440] [INSPIRE].

[68] D.L. Jafferis, I.R. Klebanov, S.S. Pufu and B.R. Safdi, Towards the F-theorem: $N=2$ field theories on the three-sphere, JHEP 06 (2011) 102 [arXiv:1103.1181] [INSPIRE].

[69] L. Fei, S. Giombi, I.R. Klebanov and G. Tarnopolsky, Generalized F-theorem and the $\epsilon$ expansion, JHEP 12 (2015) 155 [arXiv:1507.01960] [INSPIRE].

[70] S.M. Christensen and M.J. Duff, New gravitational index theorems and supertheorems, Nucl. Phys. B 154 (1979) 301 [INSPIRE]. 